\title{
Distinct-element analysis of an offshore wind turbine monopile under cyclic lateral load
}

\section{Nuo Duan PhD}

Lecturer, Institute of Foundation and Structure Technologies, Zhejiang Sci-Tech University, Hangzhou, Zhejiang, P. R. China;

formerly Department of Civil, Environmental and Geomatic Engineering,

University College London, London, UK (corresponding author:

duannuo37@hotmail.com) (Orcid:0000-0003-2585-9594)

\section{Yi Pik Cheng PhD}

Senior Lecturer, Department of Civil, Environmental and Geomatic

Engineering, University College London, London, UK

\section{Xiaomin Xu PhD}

Research Associate, Department of Engineering, University of Cambridge, Cambridge, UK

This paper presents a distinct-element method study of the dynamic behaviour of a rigid bored monopile for an offshore wind turbine foundation subject to force-controlled cyclic lateral loads. A two-dimensional model of a granular assembly was developed using the particle flow code. The model was consolidated under high gravity to simulate existing centrifuge model tests. The simulation results showed great similarity to the published experimental measurements in terms of the relationship between loading and the normalised lateral displacement. The dependency of the accumulated rotation, lateral deflection and stiffness on the two key loading characteristics, loading magnitude and direction, were analysed. Particle-scale information was employed to reveal the micromechanics of these dynamic behaviours. It was seen that relative particle displacement fields provided clear micro-scale evidence of the development of shear zones induced by the lateral cyclic loading of the pile. Meanwhile, local void densification was also observed through particle movements.

\section{Notation}

$c_{\mathrm{u}}$

$d_{1} \quad$ distance of measurement circle overlap (mm)

$d_{\text {pd }} \quad$ distance between the centre of pile and model boundary (mm)

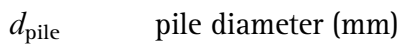

$d_{\mathrm{pp}} \quad$ distance between the centres of two adjacent pile clump particles (mm)

$d_{10} \quad 10 \%$ particle diameter $(\mathrm{mm})$

$d_{50} \quad 50 \%$ particle diameter $(\mathrm{mm})$

$d_{60} \quad 60 \%$ particle diameter $(\mathrm{mm})$

$E_{\mathrm{p}} \quad$ particle Young's modulus (Pa)

$f_{1} \quad$ first natural frequency

g gravitational acceleration $\left(\mathrm{m} / \mathrm{s}^{2}\right)$

$K_{0} \quad$ lateral stress coefficient

$k_{\mathrm{a} 1} \quad$ soil-pile unloading stiffness at first cycle $(\mathrm{N} / \mathrm{m})$

$k_{\mathrm{a} N} \quad$ soil-pile unloading stiffness at $N$ th cycle $(\mathrm{N} / \mathrm{m})$

$k_{\mathrm{b} 1} \quad$ soil-pile loading stiffness at first cycle $(\mathrm{N} / \mathrm{m})$

$k_{\mathrm{b} N} \quad$ soil-pile loading stiffness at $N$ th cycle $(\mathrm{N} / \mathrm{m})$

$k_{\mathrm{n}} \quad$ particle normal stiffness $(\mathrm{N} / \mathrm{m})$

$k_{\mathrm{s}} \quad$ particle shear stiffness $(\mathrm{N} / \mathrm{m})$

$L_{1} \quad$ distance of applied horizontal force from soil surface, eccentricity $(\mathrm{mm})$

$L_{2} \quad$ model pile penetration depth (mm)

$m_{\mathrm{d}} \quad$ radius of measurement circle $(\mathrm{mm})$

$P \quad$ lateral load (N)

$P_{\max } \quad$ maximum applied forces in cyclic loading (N)

$P_{\min } \quad$ minimum applied forces in cyclic loading $(\mathrm{N})$

$P_{\mathrm{R}} \quad$ maximum bearing capacity from the static test (N)

$R$

\begin{tabular}{|c|c|}
\hline$Y_{\max , N}$ & $\begin{array}{l}\text { displacement at maximum horizontal load for the } \\
N \text { th cycle }\end{array}$ \\
\hline$Y_{\max , 1}$ & $\begin{array}{l}\text { displacement at maximum horizontal load for the } \\
\text { 1st cycle }\end{array}$ \\
\hline$Y_{1}$ & $\begin{array}{l}\text { displacement at maximum horizontal load for the } \\
\text { 1st cycle }\end{array}$ \\
\hline$Y_{500}$ & $\begin{array}{l}\text { displacement at maximum horizontal load for the } \\
\text { 500th cycle }\end{array}$ \\
\hline$\gamma$ & bulk unit weight $\left(\mathrm{kN} / \mathrm{m}^{3}\right)$ \\
\hline$\mu$ & friction coefficient of the particles \\
\hline$\zeta_{\mathrm{b}}, \zeta_{\mathrm{c}}$ & load characteristic parameters \\
\hline
\end{tabular}

\section{Introduction}

The offshore wind farm industry is expanding rapidly in many countries. This is driven by the increasing demand for renewable, sustainable and green energy, as well as strong public support. For a typical wind turbine (e.g. $2 \mathrm{MW}$ ) in 20 40 m water depth, the tower is commonly supported by a monopile foundation, which consists of a steel tube of $4 \sim 8 \mathrm{~m}$ dia. The long-term performance of monopile foundations under wind and wave actions has therefore become increasingly important for the offshore wind turbine industry (Houlsby, 2016; Kirkwood and Haigh, 2013a).

Centrifuge modelling and $1 \mathrm{~g}$ physical modelling are effective alternatives to understand the influence of cyclic loads on monopile foundations. For example, Li et al. (2010) performed a centrifuge test on a $1 / 100$ scale monopile in sand at $100 \mathrm{~g}$ to model the behaviour of monopile foundations for offshore 
wind turbines. The homogeneous dense sand specimen had a relative density of $97 \%$. A logarithmic trend of the accumulated lateral displacement against the number of cycles, and an increase in foundation stiffness with cycling, were revealed. The logarithmic trend was proposed earlier by Long and Vanneste (1994) for full-scale slender pile tests and was also observed in $1 \boldsymbol{g}$ tests by Peralta and Achmus (2010). Kirkwood and Haigh (2013b) suggested the cyclic loading ratio of lateral loads applied to the monopile, $\zeta_{\mathrm{c}}$, which determined the direction of the cyclic loading, could change the stiffness of the soil surrounding the pile. A negative value of $\zeta_{c}$ could lead to increasing pile head displacement and rotation because a reversal in the loading direction induced a reduction of locked in stresses. Leblanc et al. (2010) performed a set of scaled monopile model tests subjected to cyclic lateral loads. The accumulation of pile rotation and change in the induced secant unloading stiffness of the system were observed, although the tests were conducted under $1 \boldsymbol{g}$ conditions. Also, the results were different from those of the centrifuge tests by $\mathrm{Li}$ et al. (2010). The accumulation of lateral displacement was assumed to follow a power law. Other model tests (such as Bhattacharya et al., 2013; Guo et al., 2015; Yu et al., 2015) also showed an increase in foundation stiffness. When this happens, the natural frequency of the whole system also increases as the Eigen frequency is proportional to the square root of stiffness.

Table 1 shows a summary of previous research, both experimental tests and numerical simulations. The $1 g$ tests could be run for a long time, but the centrifuge tests only reached up to 1000 cycles. In order to incorporate the effect of long-term cyclic loading of a pile, the concept of degradation was adopted by means of different methods. Lin and Liao (1999) and Long and Vanneste (1994) found that the degradation factor could be determined based on installation method, soil density and load ratio. They suggested a power function and a logarithmic expression, respectively. Achmus et al. (2010) suggested that the results from flexible piles fitted the logarithmic function best while the power function (such as Klinkvort (2013)) fitted the results from the rigid piles best.

Although physical modelling (e.g. centrifuge modelling and $1 \mathrm{~g}$ physical modelling) has provided fruitful results on the behaviour of monopile foundations under cyclic lateral loading, the underlying fundamental mechanisms remain unclear. Numerical modelling, especially discrete-element modelling (DEM), has been proven to provide rich insights into the micromechanics at the particle scale.

Cui and Bhattacharya $(2015,2016)$ reported DEM simulation results with loading at the mudline level, 13000 spherical particles and 1000 cycles of loading, and observed that various features, such as the increase of stiffness of granular soils under cyclic loading, the convective soil flow and the soil densification surrounding the monopile, were the main reasons underlying the increase in stiffness. Their study also pointed out that the stiffness of the surrounding soil increased with number of cycles, leading to a change in the natural frequency of the wind turbine system. Following their research, it was concluded that a 'soft-stiff' design could move the system towards the blade passing (3P) frequency. They also showed that asymmetric cyclic loading applied a larger maximum strain to soil, which resulted in a higher stress in the first cycle. It was the cyclic strain amplitude that governed the long-term dynamic stress-strain response. However, the model was relatively small, the particles were oversized, and the movement of pile was velocity controlled instead of force controlled.

In the present study, DEM simulations were performed at an acceleration of $100 \mathrm{~g}$ to compare with centrifuge model tests of a monopile under cyclic lateral loading. The dimension of the scaled monopile model was for a typical $2 \mathrm{MW}$ wind turbine. The input frequency of the cyclic loading was identified, and the influences of cyclic lateral loads on pile lateral displacement and soil stiffness were investigated.

\section{DEM modelling}

\subsection{Sample preparation}

The numerical sample for the DEM model was carefully prepared grid by grid and was subjected to a high gravitational force. It was called the GM DEM-centrifuge model in Duan and Cheng (2016). The scaling laws commonly used in centrifuge modelling were adopted in this study (Schofield, 1980). Rigid walls were used to model the boundary. The dimensions of the DEM model were $0.6 \mathrm{~m}$ in both width and depth, the same as that of Kirkwood and Haigh (2013b).

Table 1. Summary of previous studies on monopile under cyclic lateral loading

\begin{tabular}{|c|c|c|c|c|c|c|}
\hline Authors & Model & $\begin{array}{c}\text { Pile diameter, } \\
d_{\text {pile: }}: m\end{array}$ & $\begin{array}{l}\text { Load eccentricity, } \\
\qquad L_{1} / d_{\text {pile }}\end{array}$ & $\begin{array}{l}\text { Pile penetration, } \\
\qquad L_{2} / d_{\text {pile }}\end{array}$ & $\begin{array}{l}\text { Number } \\
\text { of cycles }\end{array}$ & $\begin{array}{l}\text { Loading } \\
\text { frequency: } \mathrm{Hz}\end{array}$ \\
\hline Cuéllar (2011) & $1 g$ & $7 \cdot 5$ & 4 & 4 & 5000000 & - \\
\hline Li et al. (2010) & $100 \mathrm{~g}$ & 5 & $14 \cdot 4$ & 5 & 1000 & $0.02-0.70$ \\
\hline Klinkvort (2013) & $25 \sim 125 \boldsymbol{g}$ & 2 & 15 & 6 & 500 & - \\
\hline Achmus et al. (2010) & FEM & 5 & $2 \cdot 6$ & $2 \cdot 6-5 \cdot 3$ & 10000 & - \\
\hline Kirkwood and Haigh (2013a, 2013b) & $100 \mathrm{~g}$ & 4.5 & $6 \cdot 66$ & 4.44 & 1000 & - \\
\hline
\end{tabular}




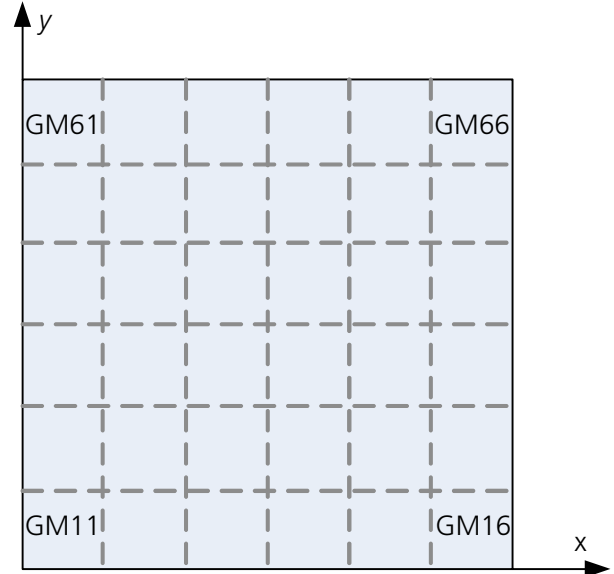

(a)

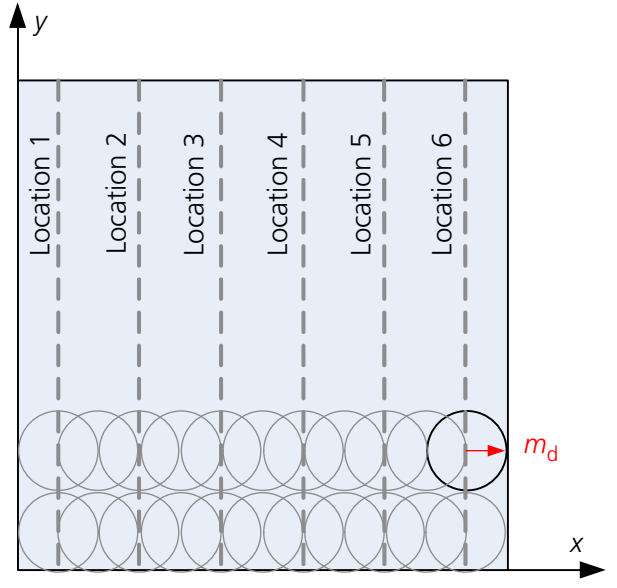

(b)

Figure 1. Schematic view of the grid method (Duan and Cheng, 2016)

In the first stage during sample generation, sand particles inside each grid were generated one by one, as illustrated in Figure 1. Approximately 280 particles were created in each grid, with an initial average porosity of $0 \cdot 25$, and the model was brought to the equilibrium state. After the bottom layer of soil was formed, all the internal walls between the grids were deleted and the locations of the surface soil particles were temporarily fixed until the layer above was successfully formed. This process was repeated until all the particles in all 36 grids were created, resulting in a total of 10080 particles in the whole model. In the second stage, a $100 \mathrm{~g}$ gravity force in the $y$ direction was applied to the whole system, and the particle flow code (PFC) model was numerically cycled again to obtain the equilibrium state. At this point the porosity had reached the final average value of $0 \cdot 185$. In the third stage, the sand particles inside the pile zone were deleted and a series of clumps was created to model the rigid monopile. The system was then cycled to an equilibrium state again with the pile in place.

The sand particles were made of discs with a maximum diameter of $7.05 \mathrm{~mm}$, a minimum diameter of $4.5 \mathrm{~mm}$, an average diameter $d_{50}=5.85 \mathrm{~mm}$ and uniformity coefficient $c_{\mathrm{u}}=d_{60} / d_{10}=1.26$ (see Figure 2). Table 2 shows the input parameters used in the DEM simulations; note the three-dimensional (3D) units presented in this paper were based on a unit depth calculation.

Figure 3(a) presents the distribution of initial void ratio in the DEM model. The average void ratio line slightly decreases with soil depth, with a variation of $0 \cdot 05$. A uniformly distributed void ratio, with a slightly lower value at the bottom, was achieved using the proposed grid method (GM). Figure 3(b) compares the average lateral and vertical stress distributions with depth. The value of lateral stress coefficient $K_{0}$ is $0 \cdot 65$.

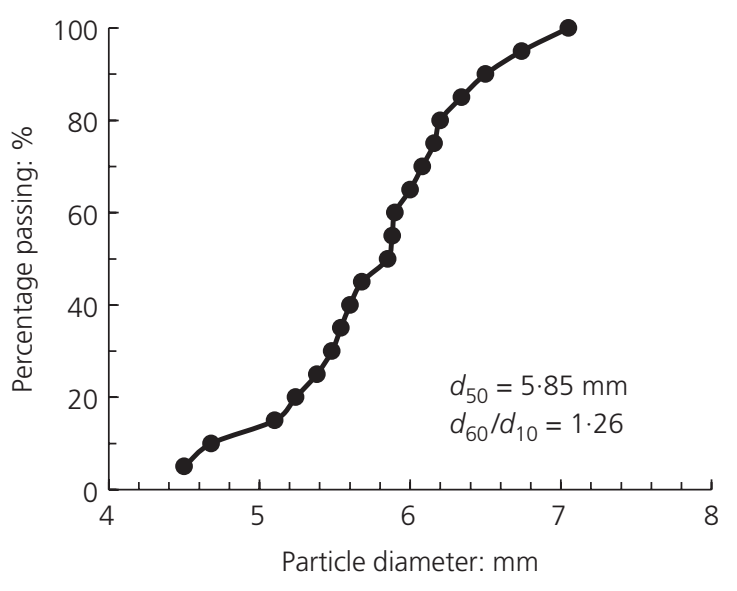

Figure 2. Particle size distribution used in DEM modelling

Table 2. Input parameters for DEM simulations

$\begin{array}{ll}\text { Density of sand particles: } \mathrm{kg} / \mathrm{m}^{3} & 2650 \\ \text { Density of clump particles for pile: } \mathrm{kg} / \mathrm{m}^{3} & 66 \cdot 65 \\ \text { Sand grain size, } d_{50}: \mathrm{mm} & 5 \cdot 85 \\ \text { Friction coefficient of soil and pile particles, } \mu & 0 \cdot 5 \\ \text { Sand particle Young's modulus, } E_{\mathrm{p}}: \mathrm{Pa} & 4 \times 10^{7} \\ \text { Contact normal stiffness of particles, } k_{\mathrm{n}}: \mathrm{N} / \mathrm{m} & 8 \times 10^{7} \\ \text { Particle stiffness ratio }\left(k_{\mathrm{s}} / k_{\mathrm{n}}\right) & 0 \cdot 25 \\ \text { Contact normal stiffness of walls, } k_{\mathrm{n}}: \mathrm{N} / \mathrm{m} & 6 \times 10^{12} \\ \text { Initial average porosity } & 0.25 \\ \text { Final average porosity (after adding gravity) } & 0 \cdot 185 \\ \text { Bulk unit weight, } \gamma \text { bulk: } \mathrm{kN} / \mathrm{m}^{3} & 2115 \cdot 3\end{array}$

\subsection{Pile installation}

The pile used in this study was rigid. It was made of 4940 small-sized particles with radius $R$ of $1.125 \mathrm{~mm}$, forming its four sides, as shown in Figure 4(a). The pile particles overlapped each other, and the distance between the centres of two 


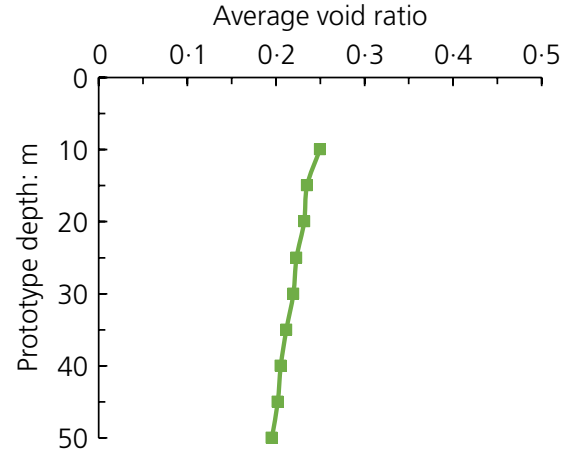

(a)

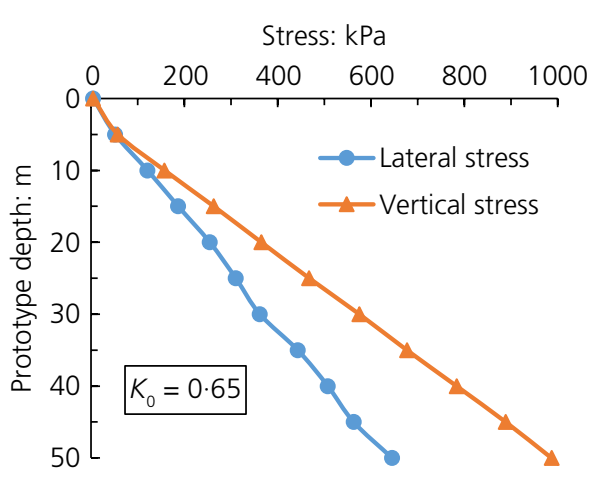

(b)

Figure 3. Soil condition at equilibrium before pile installation: (a) distribution of initial void ratio; (b) average vertical and lateral stress

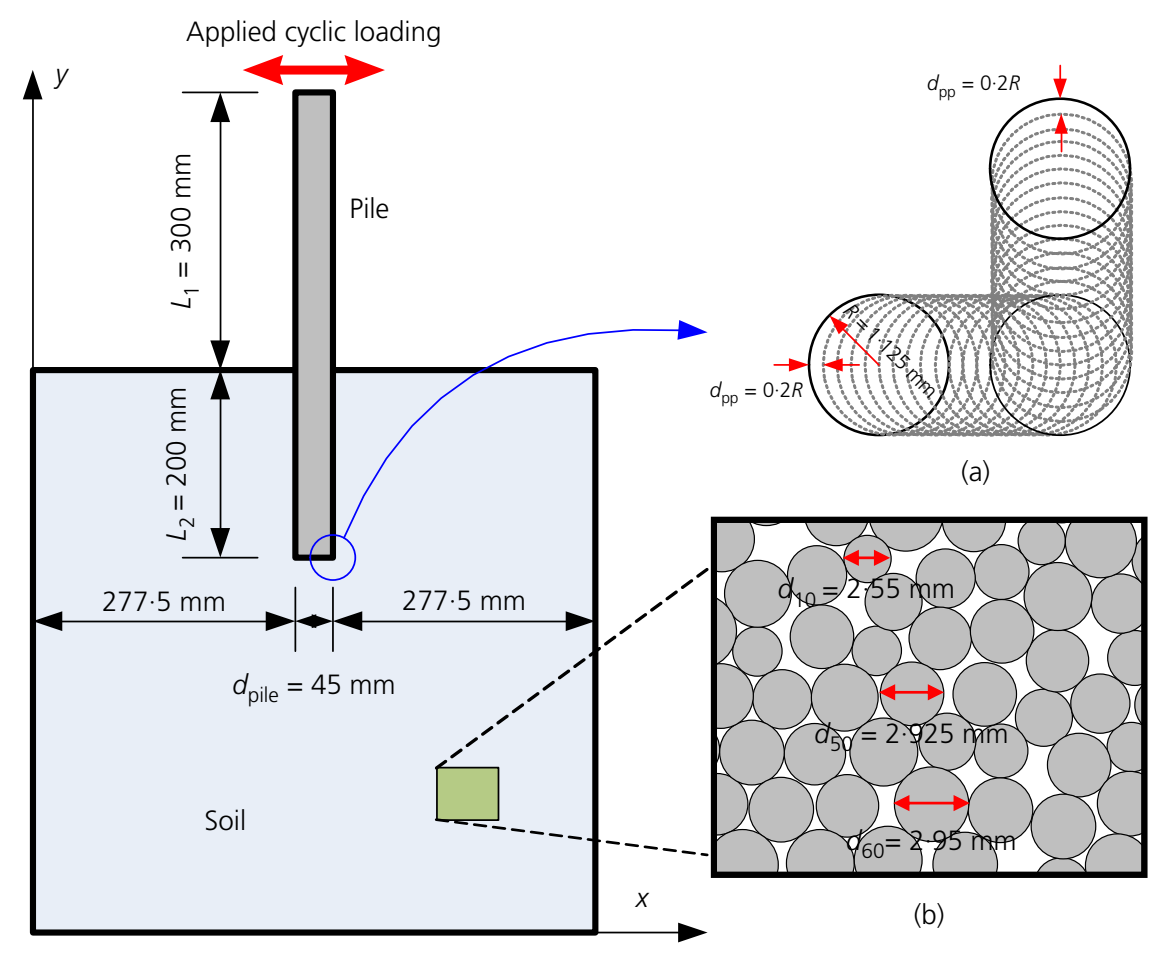

Figure 4. Schematic view of the PFC model: (a) close-up view of pile clumps; (b) a typical particle assembly at equilibrium before pile installation

adjacent particles, $d_{\mathrm{pp}}$, was $0 \cdot 2 R$. In order to satisfy the density equivalence condition, the density of the clump particles was scaled accordingly. A smooth pile surface was achieved due to the small size and the short distance between particles used.

Figure 4 also shows a sketch for the model including the location and dimensions of the pile, and a snapshot of the sand particles at the final equilibrium state. The dimensions of the DEM model followed those of the experimental set-up in centrifuge modelling. The monopile in the DEM simulations had a diameter $d_{\text {pile }}$ of $45 \mathrm{~mm}\left(\sim 8 d_{50}\right)$ and a penetration depth
$L_{2}$ of $200 \mathrm{~mm}\left(\sim 34 d_{50}\right)$; the cyclic load was applied at a loading eccentricity $L_{1}$ of $300 \mathrm{~mm}$ above the soil surface using a sine function. The direction of the first cycle was left, and the left side was treated as the passive side and the right side was the active side.

\subsection{Lateral loading characteristics}

The applied cyclic lateral loading characteristics are commonly described using two constants, $\zeta_{\mathrm{b}}$ and $\zeta_{\mathrm{c}}$, as expressed below (Klinkvort, 2013; Leblanc et al., 2010; Long and Vanneste, 1994; Rosquoet et al., 2007). 


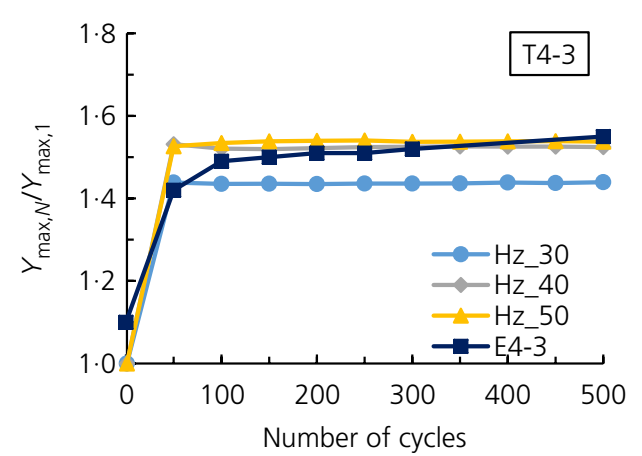

(a)

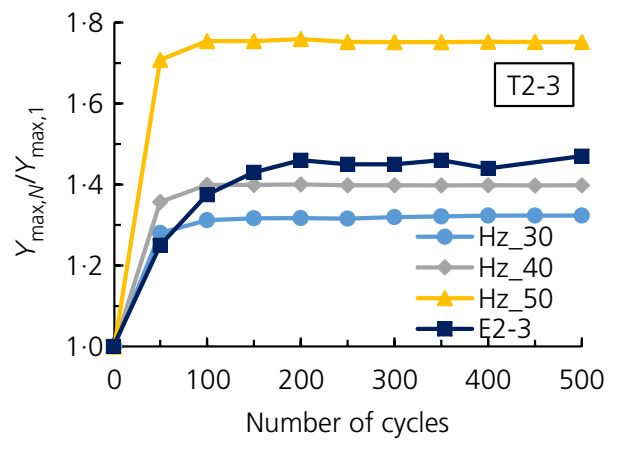

(b)

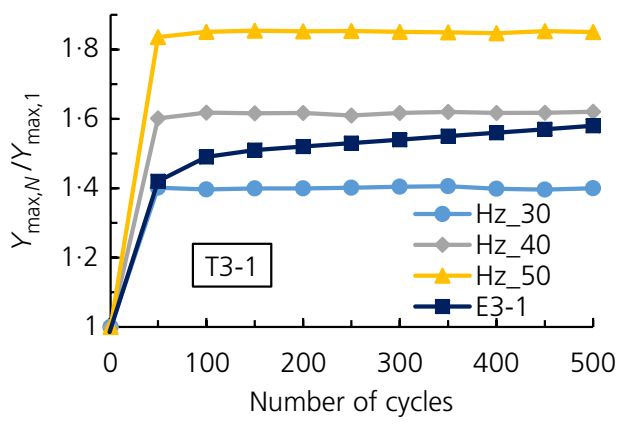

(c)

Figure 5. Comparison of normalised lateral displacement $(N=500$ relative to $N=1)$ between the centrifuge experimental and the GM-DEM model results at different loading frequencies when $\zeta_{b}=0.36$ : (a) $\zeta_{c}=0$, (b); $\zeta_{c}=0.5 ;$ (c) $\zeta_{c}=-0.5$

1. $\zeta_{\mathrm{b}}=\frac{P_{\max }}{P_{\mathrm{R}}}$

2. $\zeta_{\mathrm{c}}=\frac{P_{\min }}{P_{\max }}$

where $P_{\max }$ and $P_{\min }$ are the maximum and minimum applied cyclic load; $P_{\mathrm{R}}$ is the maximum bearing capacity from the static test, which is defined as the load corresponding to a settlement that is equal to $10 \%$ of the pile diameter $\left(P_{\mathrm{R}}=1850 \mathrm{~N}\right.$ in this study (Duan and Cheng, 2015)). $\zeta_{\mathrm{b}}$ is a normalised force parameter that describes how close the cyclic magnitudes are carried out to the static bearing capacity. For instance, when $\zeta_{\mathrm{b}}=1$, the cycles are carried out at a magnitude reaching the static bearing capacity. On the other hand, $\zeta_{c}$ describes the direction of the cyclic loading, with $\zeta_{\mathrm{c}} \geq 0$ for one-way loading and $\zeta_{\mathrm{c}}<0$ for two-way loading. When using dimensional analysis to transform results from a model to a prototype scale, some knowledge of the relevant phenomena is normally required to determine the governing parameters (Randolph, 1981). It is assumed here that there is no pore pressure build-up during the quasi-static lateral loading of the monopile. Also the cyclic lateral loading characteristics described in this paper do not consider wind and wave misalignments, even if they do not often act in the same direction. As a result, this may lead to an overprediction of foundation tilting by the DEM simulations, following the findings by Nikitas et al. (2016).

\section{Comparison with centrifuge testing results}

The long-term cyclic loading of wind turbine foundations can change the surrounding soil's stiffness, and hence the resonant frequency of the soil-pile system, which can lead to the accumulation of irreversible deformations (Cui and Bhattacharya, 2016). Offshore wind turbines are subjected to multiple loads: wind, waves, rotational frequency (1P) and blade passing frequency (3P) loads. The loads on the foundation are a combination of cyclic and dynamic loads. The wind will apply cyclic load, but the $1 \mathrm{P}$ and $3 \mathrm{P}$ will apply dynamic loads. The wave loading can be mildly dynamic or severely dynamic depending on the turbine size. The interactions of these loads could be complicated (Arany et al., 2016), but they are not considered in this paper. The typical excitation ranges are $1 \mathrm{P}(0 \cdot 18 \sim 0 \cdot 32 \mathrm{~Hz})$ and $3 \mathrm{P}(0 \cdot 5 \sim 1 \mathrm{~Hz})$. So far, offshore wind turbines have been designed with the first natural frequency, $f_{1}(0 \cdot 32 \sim 0 \cdot 5 \mathrm{~Hz})$, which is between $1 \mathrm{P}$ and 3P. In the wind industry sector this is referred to as a 'softstiff' structure. However, it is possible to design a 'soft-soft' structure with $f_{1}$ below $1 \mathrm{P}$, or a 'stiff-stiff' structure with $f_{1}$ 
above 3P. The choice of frequency range for $f_{1}$ depends on the stiffness of the foundation. In general, less steel is required for a soft structure (Arshad and O'Kelly, 2013; Cuéllar, 2011; Cui and Bhattacharya, 2015; Leblanc, 2009).

In this paper, only the impacts of the wave and wind loads were considered. For example, the frequency of the wave was around $0 \cdot 1 \mathrm{~Hz}$, and so the applied load frequency at $100 \mathrm{~g}$ was $10 \mathrm{~Hz}$ due to the scaling law. However, in centrifuge modelling, as listed in Table 1, the typical applied loading frequency ranged from $0.02 \mathrm{~Hz}$ to $0.7 \mathrm{~Hz}$ at the $100 \mathrm{~g}$ level, implying that a very low prototype frequency range was modelled. It should be noted that, in DEM modelling, the input loading frequency also greatly affects the simulation time. For example, a ten-cycle simulation of this DEM model under $0 \cdot 1 \mathrm{~Hz}$ requires a week (for $3.6 \mathrm{GHz}, 32 \mathrm{G}$ random access memory (Ram) capacity). Therefore, it is essential to find an appropriate loading frequency to ensure a reasonable and efficient modelling.

Figure 5 shows the comparison between the DEM model and centrifuge experimental results at three different loading frequencies (i.e. $30 \mathrm{~Hz}, 40 \mathrm{~Hz}$ and $50 \mathrm{~Hz}$ ). The magnitude of $\zeta_{\mathrm{b}}$ is fixed at 0.36 , and the value of $\zeta_{\mathrm{c}}$ is varied from -0.5 to 0.5 . For each DEM modelling, the normalised lateral displacement $Y_{\max , N} / Y_{\max , 1}$ was extracted, and then compared with that of the centrifuge modelling results from Klinkvort (2013). It can be seen from the figures that the DEM simulation with $40 \mathrm{~Hz}$ loading frequency matched best with that of the centrifuge experimental data. Therefore, the loading frequency of $40 \mathrm{~Hz}$ was chosen for all the following DEM simulations in this study.

Figure 6 presents another comparison between the DEM modelling results and centrifuge modelling results at $\zeta_{c}=-0 \cdot 5$, with a loading frequency of $40 \mathrm{~Hz}$. For small values of $\zeta_{\mathrm{b}}$ (e.g. $\zeta_{\mathrm{b}}=0.08$, as shown in Figure 6(a)), the DEM results give a lower prediction than those of the centrifuge modelling (E1-4). However, the differences between the DEM modelling and centrifuge modelling become much smaller when $\zeta_{\mathrm{b}}$ ranges from $0 \cdot 18$ to $0 \cdot 36$. The normalised accumulated lateral displacement obtained from both the DEM tests and the centrifuge experiments matches well, as shown in Figures 6(b) and $6(\mathrm{c})$. Hence, a larger $\zeta_{\mathrm{b}}$ value results in a better comparison between the two-dimensional (2D) simulation and the 3D centrifuge tests. It was found that the first ten cycles induced the most significant amount of normalised displacement. This phenomenon was seen both in the centrifuge model data and the DEM simulation data. In total, 15 different cases with various loading characteristics were analysed, as listed in Tables 3 and 4.

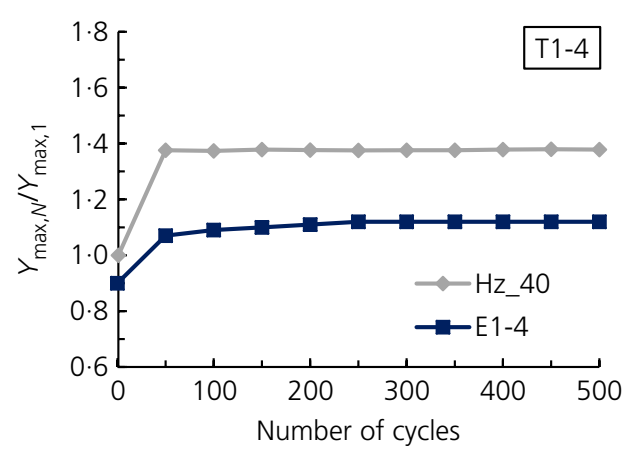

(a)

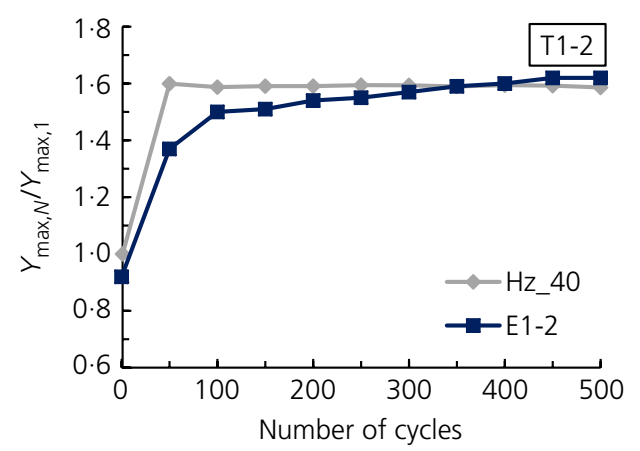

(b)

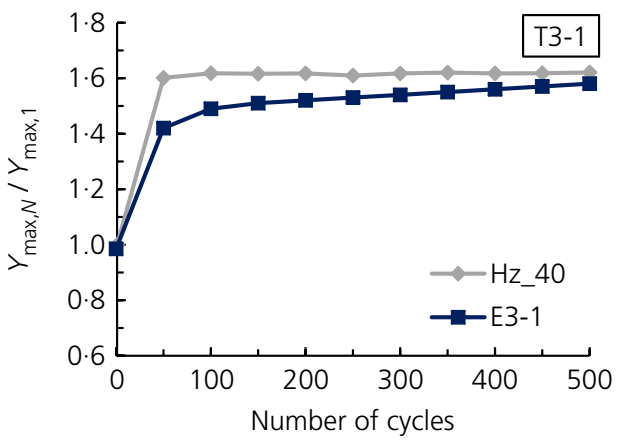

(c)

Figure 6. Comparison of the normalised lateral displacement $(N=500$ relative to $N=1)$ between the centrifuge experimental results and the GM-DEM model results when $\zeta_{c}=-0 \cdot 5$ : (a) $\zeta_{b}=0 \cdot 08 ;(b) \zeta_{b}=0 \cdot 18 ;(c) \zeta_{b}=0.36$ 
Table 3. Loading cases of centrifuge experimental tests (Klinkvort, 2013)

\begin{tabular}{llll} 
Experiment & \multicolumn{1}{c}{$\boldsymbol{\zeta}_{\mathbf{b}}$} & $\boldsymbol{\zeta}_{\mathbf{c}}$ & $\begin{array}{l}\text { Diameter of pile: } \\
\mathbf{m m}\end{array}$ \\
\hline E1-2 & 0.18 & $-0.46 \sim-0.32$ & 28 \\
E1-3 & 0.36 & $-0.46 \sim-0.32$ & 28 \\
E1-4 & 0.08 & $-0.46 \sim-0.32$ & 28 \\
E2-3 & $0.25 \sim 0.29$ & 0.54 & 40 \\
E3-1 & $0.33 \sim 0.34$ & -0.5 & 40 \\
E4-3 & $0.15 \sim 0.36$ & 0.05 & 40 \\
\end{tabular}

Table 4. Loading cases of the DEM GM-centrifuge simulations

\begin{tabular}{|c|c|c|c|c|}
\hline DEM & $\zeta_{\mathrm{b}}$ & $\zeta_{\mathrm{c}}$ & $Y_{1}: \mathrm{mm}$ & $Y_{500}: \mathrm{mm}$ \\
\hline T1-2 & $0 \cdot 18$ & -0.5 & 0.4393 & 0.6970 \\
\hline Т1-3 & 0.36 & -0.37 & 0.9326 & 1.4648 \\
\hline T1-4 & 0.08 & -0.5 & 0.2095 & 0.2887 \\
\hline T2-1 & $0 \cdot 3$ & -0.8 & 0.7213 & $1 \cdot 1719$ \\
\hline $\mathrm{T} 2-2$ & 0.3 & -0.9 & 0.7166 & 1.1358 \\
\hline$T 2-3$ & 0.36 & 0.5 & 1.0215 & 1.4194 \\
\hline T3-1 & 0.36 & -0.5 & 0.9061 & $1 \cdot 4448$ \\
\hline T4-3 & 0.36 & 0 & 0.9711 & 1.4802 \\
\hline T5-1 & $0 \cdot 3$ & 0 & $0 \cdot 8300$ & $1 \cdot 1800$ \\
\hline T5-2 & 0.4 & 0 & 1.0500 & 1.6700 \\
\hline T5-3 & 0.5 & 0 & 1.3300 & $2 \cdot 2600$ \\
\hline T5-4 & 0.6 & 0 & $1 \cdot 5900$ & $2 \cdot 7400$ \\
\hline T6-2 & 0.5 & -0.5 & $1 \cdot 2400$ & $2 \cdot 1900$ \\
\hline T6-3 & 0.5 & $-0 \cdot 37$ & $1 \cdot 2500$ & $2 \cdot 1700$ \\
\hline T6-4 & 0.5 & 0.5 & 1.4000 & $2 \cdot 2000$ \\
\hline
\end{tabular}

\section{Results and discussion}

\subsection{The effect of loading characteristics}

To highlight the influence of loading characteristics, Figures 7 and 8 present a comparison of normalised lateral displacement for different values of $\zeta_{\mathrm{b}}$ and $\zeta_{\mathrm{c}}$, respectively. It can be seen from Figure 7 that, for both cases with $\zeta_{\mathrm{c}}=-0.5$ and $\zeta_{\mathrm{c}}=0$, as $\zeta_{\mathrm{b}}$ increases from $0 \cdot 08$ to $0 \cdot 5$, the normalised accumulated displacement increases. There is only a very limited increase of the normalised lateral displacement when the value of $\zeta_{\mathrm{b}}$ increases from $0 \cdot 5$ to $0 \cdot 6$. This is most likely due to the limitations in soil densification or a boundary effect. When $\zeta_{\mathrm{b}}$ is above $0 \cdot 6$, it is considered a very large loading amplitude within the normal service time.

Figures 8 (a) and 8 (b) shows all the simulations under the same $\zeta_{\mathrm{b}}=0.36$ and $\zeta_{\mathrm{b}}=0.5$, respectively. Both figures show very similar phenomena. The results show that when $\zeta_{\mathrm{c}}$ is $-0 \cdot 5$, the two-way loading results in the greatest pile head displacement, whereas when $\zeta_{\mathrm{c}}$ is positive at $0 \cdot 5$, the one-way loading results in the smallest pile head displacement. This means that, for any two-way test with the same $\zeta_{\mathrm{b}}$, the displacement increases with the absolute value of $\zeta_{c}$, and for any one-way loading with the same $\zeta_{\mathrm{b}}$, the displacement decreases with increasing $\zeta_{\mathrm{c}}$. Hence, the loading direction $\zeta_{\mathrm{c}}$ substantially controls the magnitude of pile head displacement. Both Figures 7 and 8 present a phenomenon whereby the principle lateral displacement occurs in the first ten cycles, for all cases, and then tends not to vary afterwards. This characteristic was also observed by Chen et al. (2015).

Figure 9 summarises the influences of the two loading characteristics, $\zeta_{\mathrm{c}}$ and $\zeta_{\mathrm{b}}$, on the normalised lateral displacement. It can be observed from Figure 9(a) that $Y_{\max , N} / Y_{\max , 1}$ generally increases as $\zeta_{\mathrm{b}}$ increases. This trend is in line with the published data from centrifuge modelling. The effect of $\zeta_{\mathrm{c}}$, on the other hand, is more complex. Interestingly, both the DEM results and the centrifuge data show very similar trends, although with different transition points. In general, the DEM modelling data obtained are comparable with those from the centrifuge modelling.

\subsection{Accumulated lateral displacements related to two-way loading}

Figure 10 presents the first ten cycles of three cases of lateral force-displacement curves of the monopile under different directions of cyclic lateral loads $\left(\zeta_{\mathrm{c}}=-0 \cdot 5,0\right.$ and $\left.0 \cdot 5\right)$ for the same amplitude $\left(\zeta_{\mathrm{b}}=0 \cdot 38\right)$. The lateral displacements of the pile were measured at the pile head. In Figure 10(a) when $\zeta_{\mathrm{c}}$ is

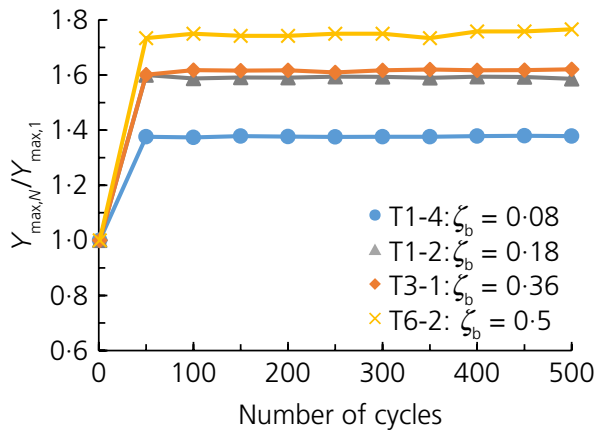

(a)

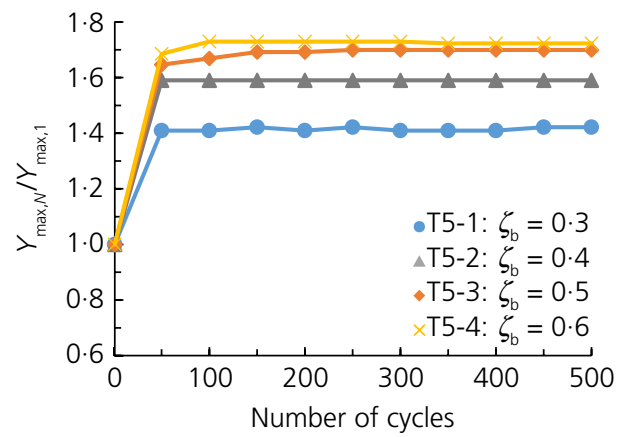

(b)

Figure 7. Effect of $\zeta_{b}$ on the normalised lateral displacement $(N=500$ relative to $N=1)$ : (a) $\zeta_{c}=-0 \cdot 5$; (b) $\zeta_{c}=0$ 


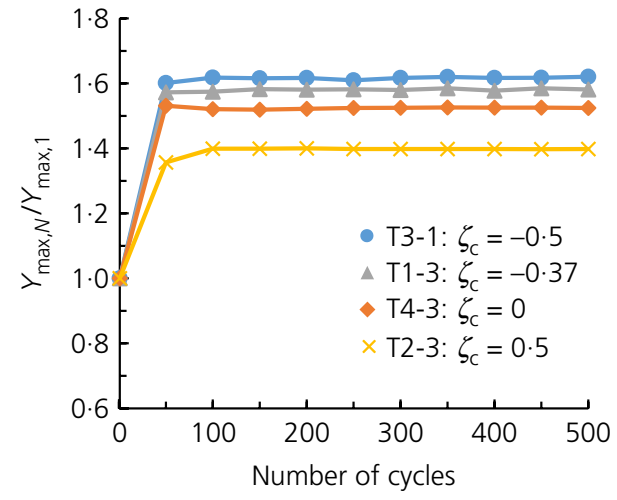

(a)

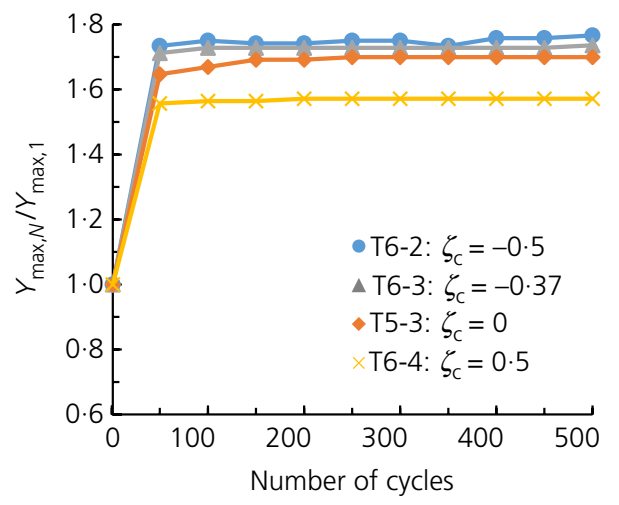

(b)

Figure 8. Effect of $\zeta_{c}$ on the normalised lateral displacement $(N=500$ relative to $N=1)$ : (a) $\zeta_{b}=0.36$; (b) $\zeta_{b}=0.5$

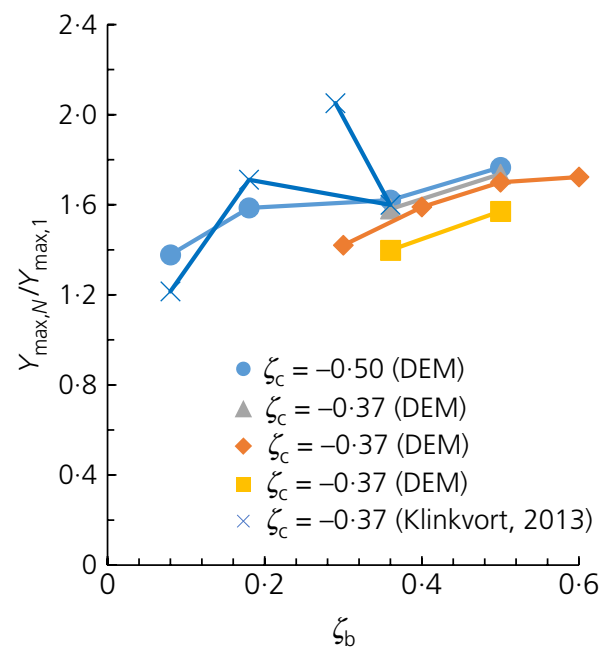

(a)

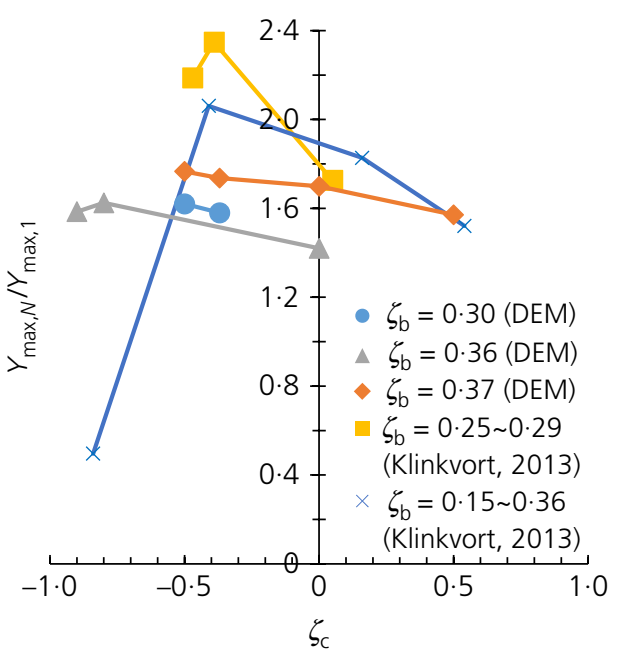

(b)

Figure 9. Effect of $(\mathrm{a}) \zeta_{\mathrm{b}}$ and $(\mathrm{b}) \zeta_{\mathrm{c}}$ on the normalised lateral displacement $(N=500$ relative to $N=1)$

negative, implying a two-way loading test, larger accumulated lateral displacements are seen compared to the one-way tests (see Figures 10(b) and 10(c)). In Figure 10, the unloading stiffnesses in the first and the tenth cycle are marked with $k_{\mathrm{a} 1}$ and $k_{\mathrm{a} 10}$, and their actual values are shown in Table 5. It is clear from Figure 10 and Table 5 that $k_{\mathrm{a} 10}$ is always smaller than $k_{\mathrm{a} 1}$ in any of the three cases. This implies that the foundation moves slightly more within each cycle (when unloading stiffness reduces) with an increasing number of cycles. The extent of this stiffness reduction becomes most significant in the oneway loading case $\left(k_{\mathrm{a} 10} / k_{\mathrm{a} 1}=25 \%\right.$ when $\left.\zeta_{\mathrm{c}}=0 \cdot 5\right)$, which is mainly controlled by the initially high unloading stiffness value in the first cycle when $\zeta_{\mathrm{c}}$ is positive.

In general, the loading and unloading in the first cycle exhibits evident soil non-linearity, so the loading secant stiffness reduces with increasing lateral displacement in the first cycle.
These loading stiffnesses are, however, an indication of the foundation stiffness, and they are marked as $k_{\mathrm{b} 1}$ and $k_{\mathrm{b} 10}$ in Figure 10. For the same applied loading amplitude $\left(\zeta_{\mathrm{b}}=0 \cdot 38\right)$, Table 6 shows that the initial foundation stiffnesses $k_{\mathrm{b} 1}$ of all the three cases are similar, ranging from 1.02 to 1.49 . But the foundation stiffnesses after ten cycles $k_{\mathrm{b} 10}$ increase in all three cases, with the greatest increase also in the one-way loading case when $\zeta_{\mathrm{c}}$ is positive $\left(k_{\mathrm{b} 10} / k_{\mathrm{b} 1}=339 \%\right)$. Clearly, the soil-pile interactions of all three cases induce a reduction in the porosity of the soil (the soil densifies) with increasing number of cycles, hence the foundation stiffness increases and the incremental accumulation of permanent displacement reduces, as was shown by Cui and Bhattacharya (2016). Accordingly, the natural frequency of the system increases as the Eigen frequency is proportional to the square root of stiffness (Bhattacharya et al., 2013; Guo et al., 2015; Yu et al., 2015). 


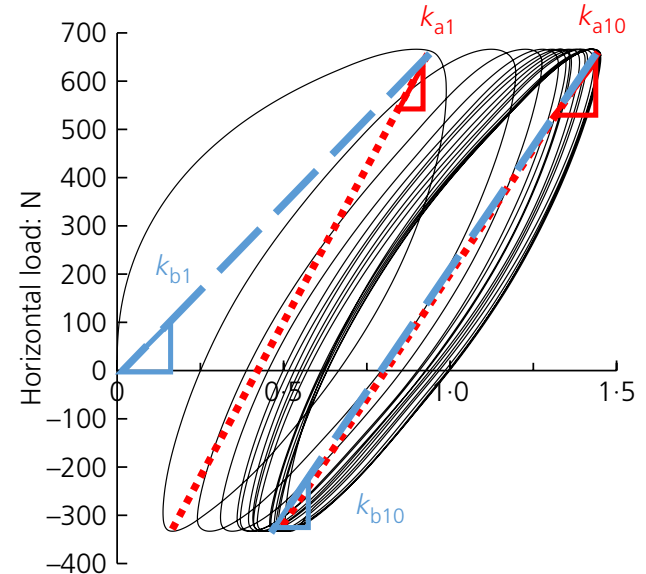

Displacement: $\mathrm{mm}$

(a)

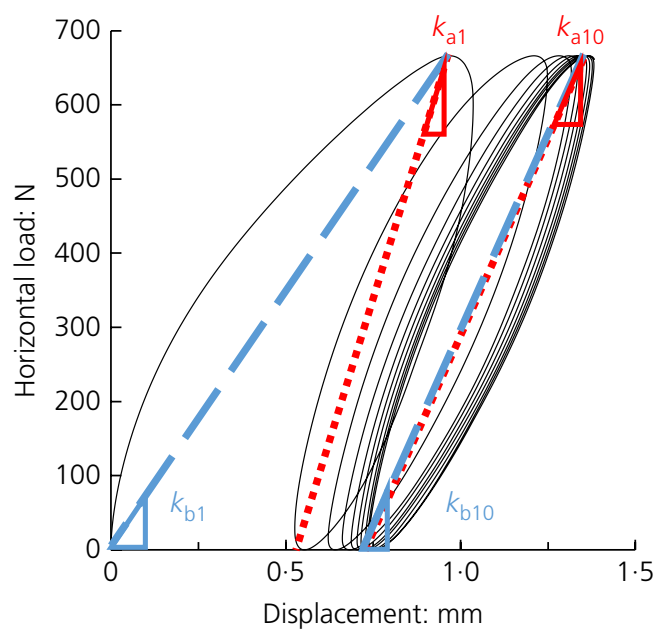

(b)

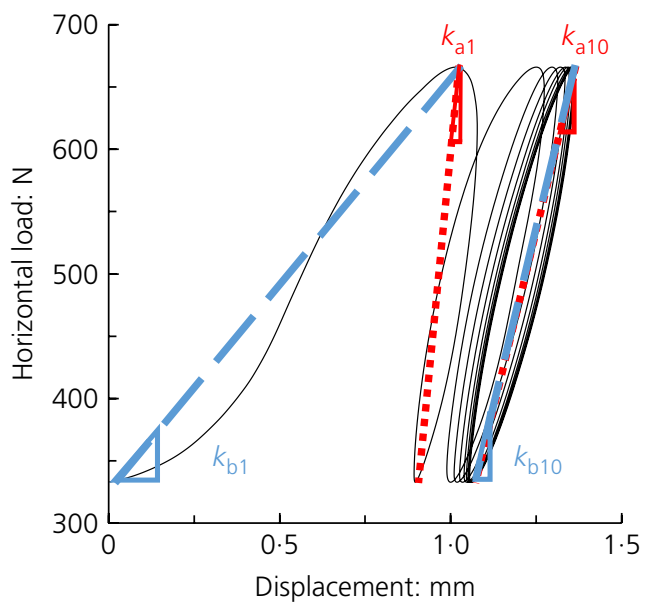

(c)

Figure 10. Pile head lateral load-displacement responses with $\zeta_{b}=0.36$ during the first ten cyclic lateral loadings: $(a) T 3-1, \zeta_{c}=-0.5$ (maximum displacement $1.404 \mathrm{~mm}$ ); (b) T4-3, $\zeta_{c}=0$ (maximum displacement $1.384 \mathrm{~mm}$ ); (c) T2-3, $\zeta_{c}=0.5$ (maximum displacement $1.366 \mathrm{~mm})$

Table 5. Unloading stiffness $k_{\mathrm{a} 1}$ and $k_{\mathrm{a} 10}$ of selected model tests

$\begin{array}{lccccl}\text { DEM } & \boldsymbol{\zeta}_{\mathbf{b}} & \boldsymbol{\zeta}_{\mathbf{c}} & \boldsymbol{k}_{\mathrm{a} 1} & \boldsymbol{k}_{\mathrm{a} 10} & \boldsymbol{k}_{\mathrm{a} 10} / \boldsymbol{k}_{\mathrm{a} 1}: \% \\ \mathrm{~T} 3-1 & 0.36 & -0.5 & 1.81 & 1.52 & 84 \\ \text { T4-3 } & 0.36 & 0 & 3.20 & 1.75 & 55 \\ \text { T2-3 } & 0.36 & 0.5 & 10.48 & 2.67 & 25\end{array}$

Table 5 also shows that the unloading stiffness gradient $k_{\mathrm{a} 1}$ is relatively small when the loading direction is two-way $\left(\zeta_{c}=-0 \cdot 5\right)$. As the number of loading cycles increases, there is a gradual accumulation of both the maximum loading displacement (cyclic maxima) and the minimum unloading displacement (cyclic minima). The unloading stiffness $k_{\mathrm{a} N}$ continues to reduce slightly from its first cycle. However, these accumulations of displacement and changes in stiffness are not as dominant as those induced by the direction of loading, that is,
Table 6. Foundation stiffness $k_{\mathrm{b} 1}$ and $k_{\mathrm{b} 10}$ of selected model tests

\begin{tabular}{cccccc} 
DEM & $\boldsymbol{\zeta}_{\mathbf{b}}$ & $\boldsymbol{\zeta}_{\mathbf{c}}$ & $\boldsymbol{k}_{\mathrm{b} \mathbf{1}}$ & $\boldsymbol{k}_{\mathrm{b} 10}$ & $\boldsymbol{k}_{\mathrm{b} 10} / \boldsymbol{k}_{\mathbf{b} 1}: \%$ \\
\hline T3-1 & 0.36 & -0.5 & 1.02 & 1.52 & 149 \\
T4-3 & 0.36 & 0 & 1.49 & 2.36 & 158 \\
T2-3 & 0.36 & 0.5 & 1.31 & 4.44 & 339
\end{tabular}

what happened in the first cycle. For example in test T3-1, the first unloading from 666 to $-333 \mathrm{~N}$ created a recoil of $0.79 \mathrm{~mm}$ and the final unloading created a recoil of $1.12 \mathrm{~mm}$ in the tenth cycle. It may be seen in Figure 10(b) that the first reloading induced $0.4 \mathrm{~mm}$ lateral displacement from 0 to $666 \mathrm{~N}$ and the final unloading created a recoil of $0.606 \mathrm{~mm}$ in the tenth cycle. In Figure 10(c), the first reloading induced only $0.146 \mathrm{~mm}$ lateral displacement from 0 to $666 \mathrm{~N}$ and the final unloading created a recoil of $0.273 \mathrm{~mm}$ in the tenth cycle. 
From Table 5, when $\zeta_{\mathrm{c}}$ increases, both $k_{\mathrm{a} 1}$ and $k_{\mathrm{a} N}$ increase Nonetheless, the values of $k_{N} / k_{1}$ decrease dramatically, which means that the change of stiffness is more obvious as long as the loading direction changes from two-way into one-way $\left(\zeta_{\mathrm{c}}\right.$ increases).

\subsection{Evolution of unloading secant stiffness ratio}

Further comparisons of all cases of $k_{\mathrm{a} 500} / k_{\mathrm{a} 1}$ are shown in Figure 11. Figure 11(a) shows the impact of changes in $\zeta_{\mathrm{b}}$, whereas Figure 11(b) shows the impact of changes in $\zeta_{\mathrm{c}}$, and Klinkvort (2013) data are also shown in the figures for comparison. In Figure 11(a), although Klinkvort (2013) shows that $k_{\mathrm{a} 500} / k_{\mathrm{a} 1}$ increases as $\zeta_{\mathrm{b}}$ increases, this increase is not that obvious when all the DEM simulation cases are plotted together in the same figure. In Figure 11(a), when $\zeta_{\mathrm{c}}$ is positive $(=0 \cdot 5)$, the increase of $\zeta_{\mathrm{b}}$ causes an enhancement of the stiffness ratio. However, when $\zeta_{\mathrm{c}}=0$ with the loading characteristic still one-way, the stiffness ratio decreases a little with an increase of $\zeta_{\mathrm{b}}$. When $\zeta_{\mathrm{c}}<0$ with the loading characteristic two-way, the stiffness ratio fluctuates. The DEM data appear to be different from what was observed by Klinkvort (2013). The differences can be seen more clearly in Figure 11(b). When $\zeta_{\mathrm{c}}<0$, the stiffness ratio generally drops with the increase of $\zeta_{c}$, which is consistent with Klinkvort (2013) experimental data. However, when $\zeta_{\mathrm{c}}>0$, the changes of the stiffness ratio seem to be related more to the magnitude of loading, $\zeta_{\mathrm{b}}$. Figure 10 and Table 5 indicate that, in all cases, the unloading stiffness reduces with the number of cycles, and it is believed that the stiffness $k_{\mathrm{a} 1}$ controls the stiffness ratio.

\subsection{Particle-scale observations}

Particle-scale information was extracted from the DEM simulations, to explore the fundamental mechanisms of the different behaviour of monopiles under various loading characteristics.

\subsubsection{Effect of $\zeta_{b}$}

Figure 12 compares the accumulated particle displacement fields, with $\zeta_{\mathrm{b}}$ ranging from 0.08 to $0.50 \quad\left(\zeta_{\mathrm{c}}=-0.50\right)$. In these figures, positive displacements mean particles moving to the right. For each case, the particle displacement fields at the first cycle (see Figures 12(a)-12(d)), the 500th cycle (see Figures 12(e)-12(h)) and the normalised particle displacement, namely, the 500th particle displacement divided by first (see Figures 12(i)-12(1)), are plotted. Note that the legend scale for the first two cases (case $\mathrm{A}$ : $\zeta_{\mathrm{b}}=0.08$ and case $\mathrm{B}$ : $\zeta_{\mathrm{b}}=0 \cdot 18$, as described in Table 4) is ten times smaller than that of the last two cases (case $\mathrm{C}: \zeta_{\mathrm{b}}=0.36$ and case $\mathrm{D}: \zeta_{\mathrm{b}}=0.50$, as described in Table 4).

For all the four cases presented here, the soil influencing zones are almost of the same conical shape near the pile head, with a relatively larger area on the passive side than on the active. The influence of $\zeta_{\mathrm{b}}$ on the particle displacement field is evident from these figures. In the first cycle, the magnitude of the minimum left model scale particle displacements at the pile head of case A (see Figure 12(a)) and B (see Figure 12(b)) are $0.079 \mathrm{~mm}$ and $0.123 \mathrm{~mm}$, respectively. Taking the cut-off particle displacement as $0.002 \mathrm{~mm}$ in these two cases, their influencing zones on the passive side are at about $1 \cdot 35 d_{\text {pile }}$ and $3 \cdot 35 d_{\text {pile. }}$ Meanwhile, the maximum right particle displacements at the toe of the pile are $0.022 \mathrm{~mm}$ and $0.054 \mathrm{~mm}$, respectively. Very similar phenomena can also be observed in the other two cases, case $\mathrm{C}$ and case $\mathrm{D}$, where the minimum left particle displacements at the pile head are $0.246 \mathrm{~mm}$ and $0.337 \mathrm{~mm}$, respectively.

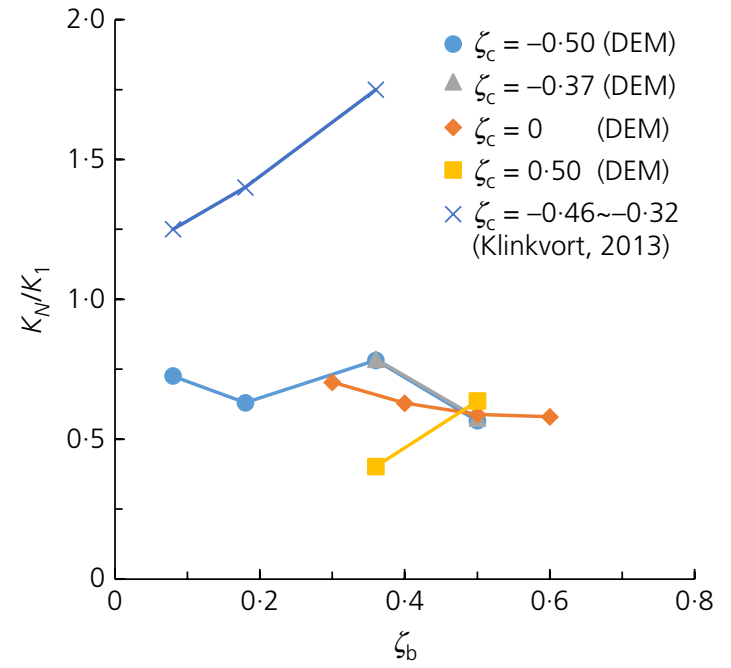

(a)

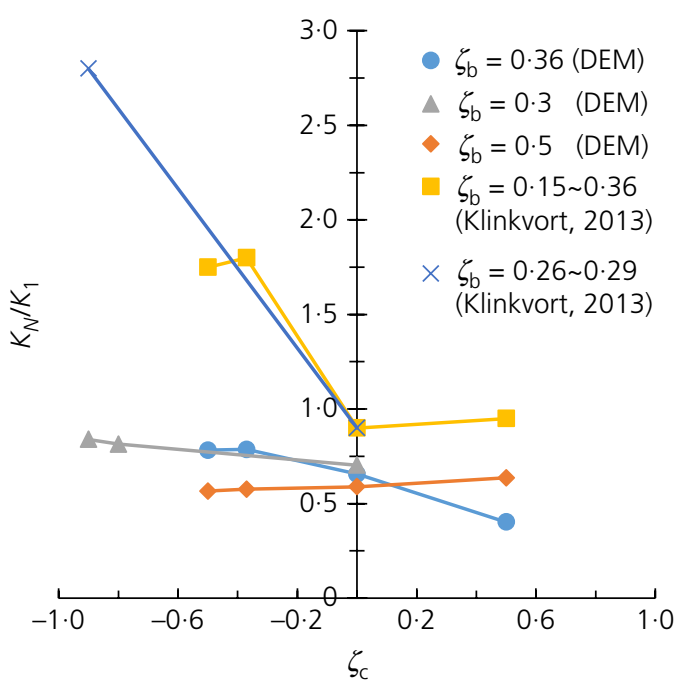

(b)

Figure 11. Effect of $(\mathrm{a}) \zeta_{\mathrm{b}}$ and $(\mathrm{b}) \zeta_{\mathrm{c}}$ on stiffness ratio $(N=500$ relative to $N=1)$ 


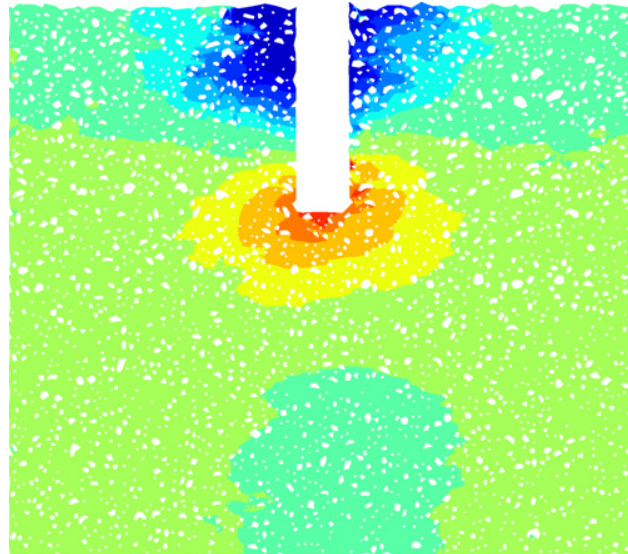

(a)

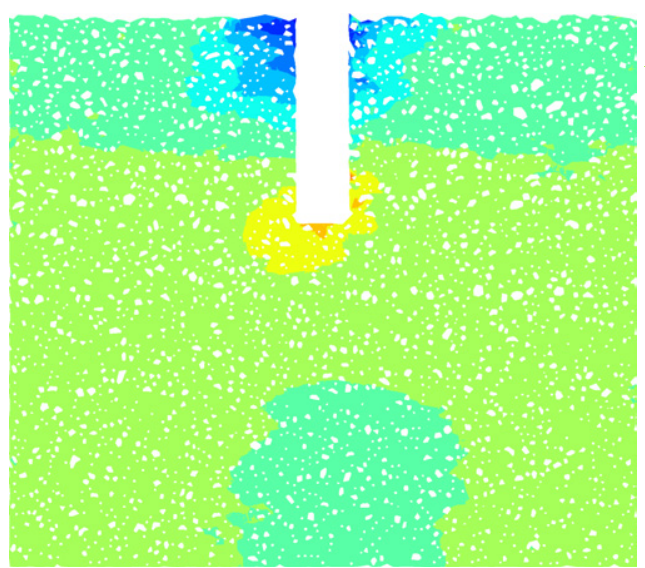

(c)

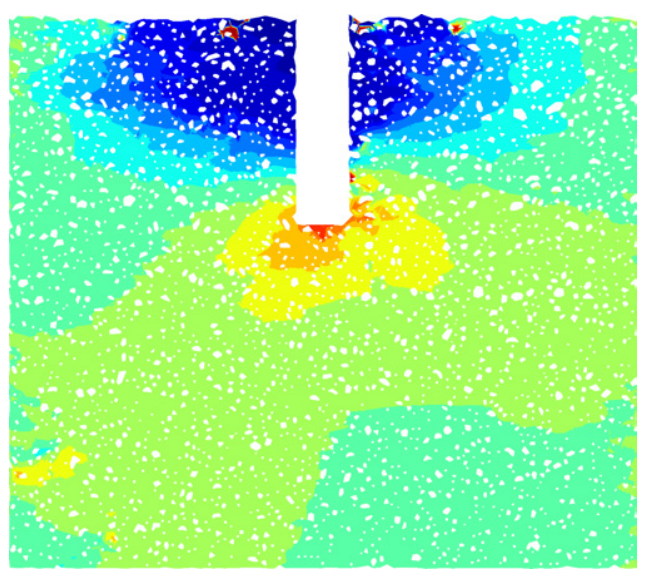

(e)

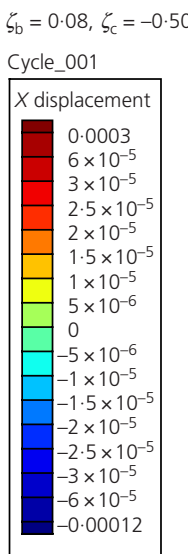

00012
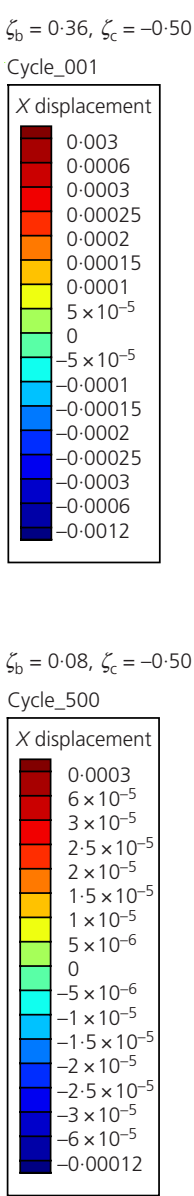

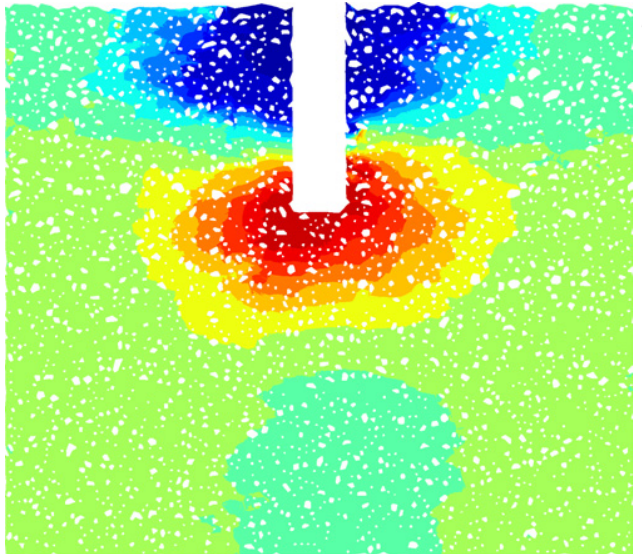

(b)
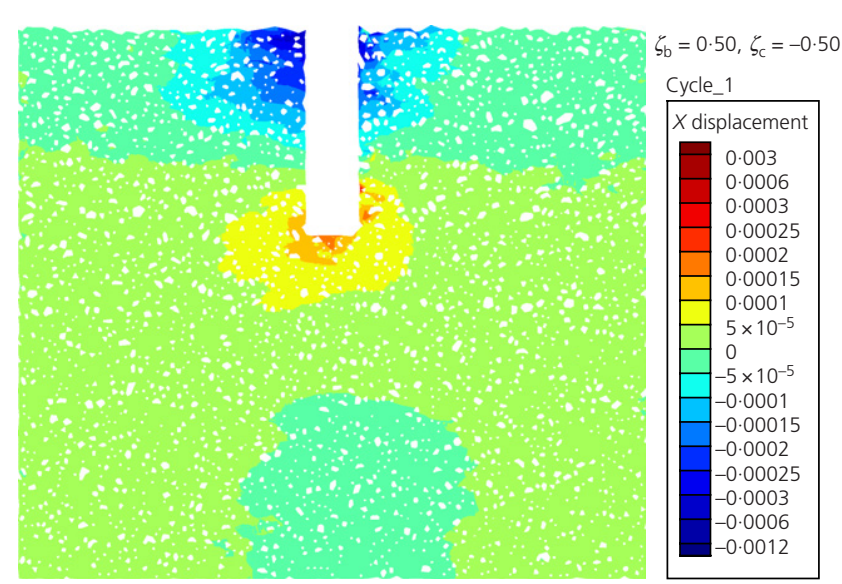

(d)
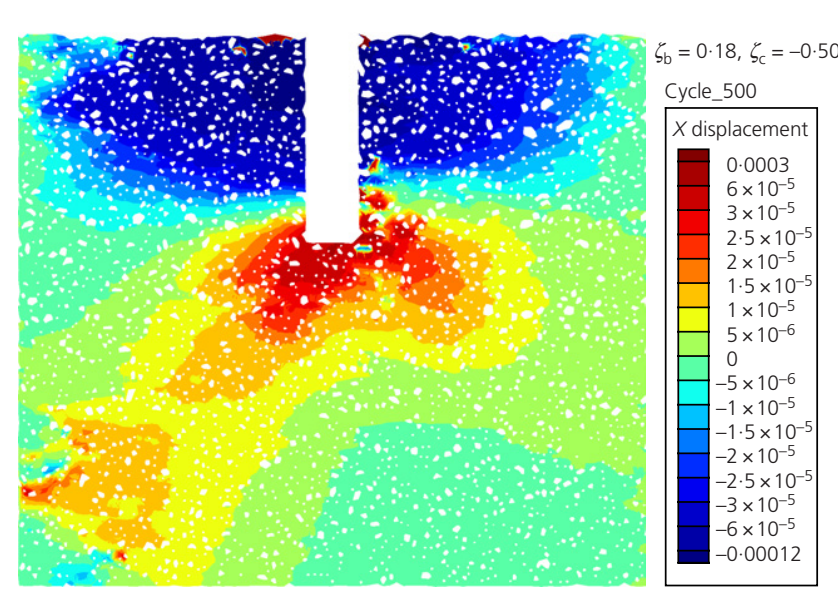

$(f)$

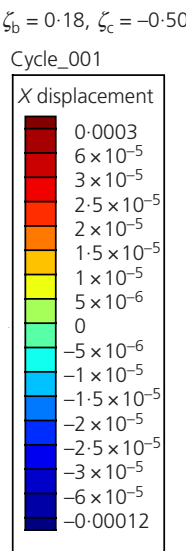

Figure 12. Effect of $\zeta_{\mathrm{b}}$ on the accumulated particle horizontal displacement field $\left(\zeta_{\mathrm{c}}=-0.50\right):(\mathrm{a}) \zeta_{\mathrm{b}}=0.08(N=1)$;

(b) $\zeta_{\mathrm{b}}=0.18(N=1) ;(\mathrm{c}) \zeta_{\mathrm{b}}=0.36(N=1) ;(\mathrm{d}) \zeta_{\mathrm{b}}=0.50(N=1) ;(\mathrm{e}) \zeta_{\mathrm{b}}=0.08(N=500) ;(\mathrm{f}) \zeta_{\mathrm{b}}=0.18(N=500) ;(\mathrm{g}) \zeta_{\mathrm{b}}=0.36(N=500) ;(\mathrm{h})$ $\zeta_{\mathrm{b}}=0.50(N=500) ;$ (i) $\zeta_{\mathrm{b}}=0.08$ (normalised); (j) $\zeta_{\mathrm{b}}=0.18$ (normalised); $(\mathrm{k}) \zeta_{\mathrm{b}}=0.36$ (normalised); (I) $\zeta_{\mathrm{b}}=0.50$ (normalised) (continued on next page)

As the number of cycles increases, the cyclic loading effect is gradually accumulated. In the 500th cycle, the area of the influencing zones is almost doubled, compared to that of the first cycle, as highlighted in Figures 12(e)-12(h). The minimum left particle displacements at the pile head are $0.089 \mathrm{~mm}, 0.215 \mathrm{~mm}$, $0.399 \mathrm{~mm}$ and $0.584 \mathrm{~mm}$, for cases A, B, C and D, respectively. 


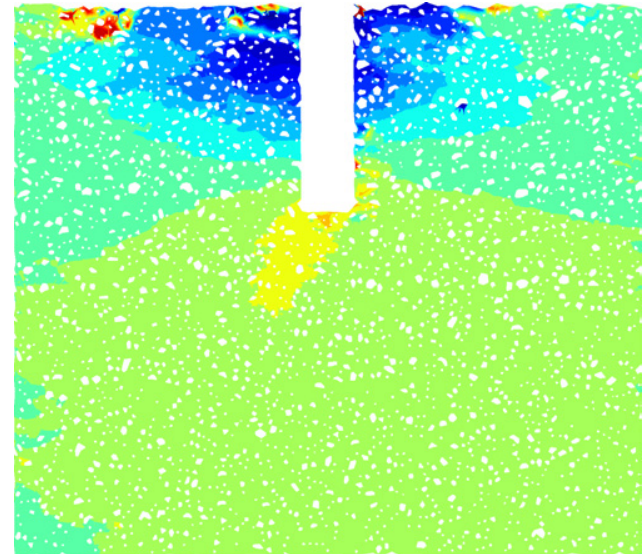

(g)

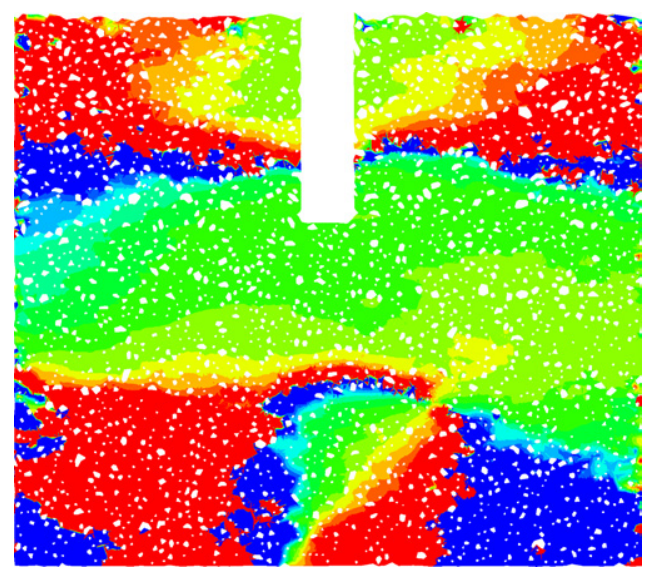

(i)

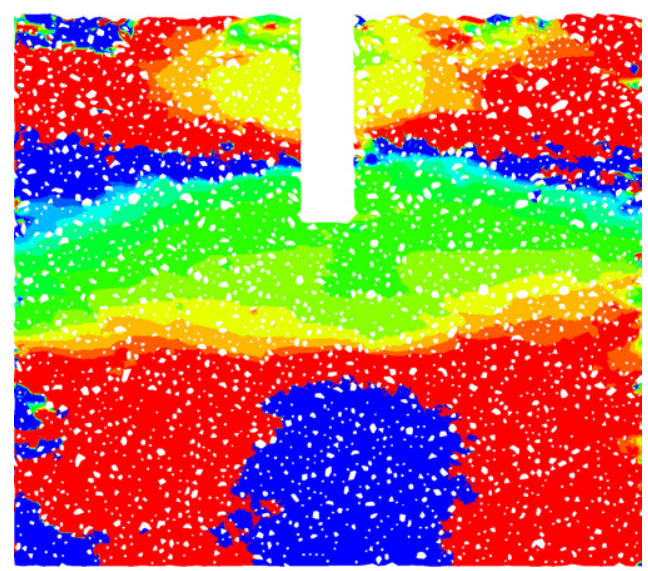

(k)

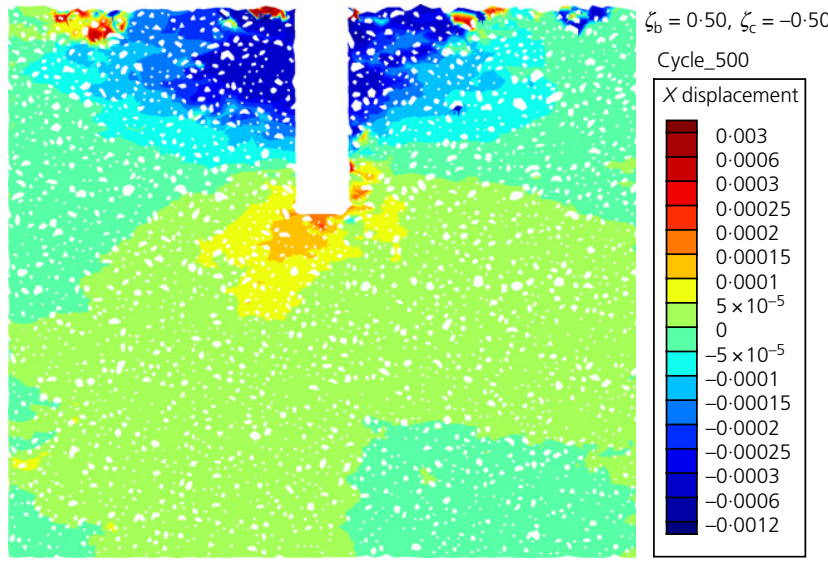

(h)

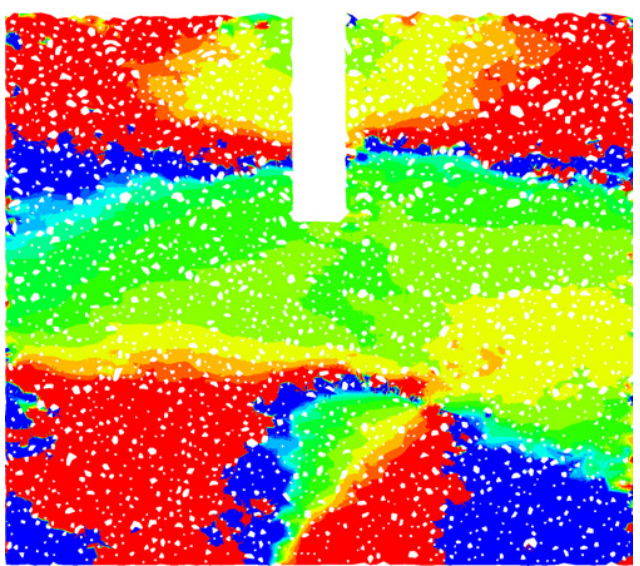

(j)

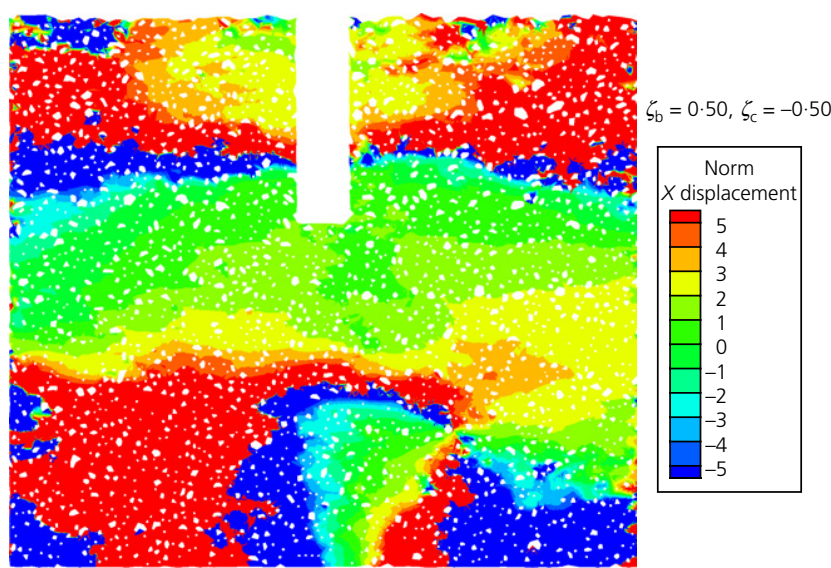

(l)

Figure 12. Continued

This is further elaborated in Figures 12(i)-12(1), where the normalised particle displacements are plotted. The location of the 'zero' value boundary for the first cycle is horizontal, but it changes to a sloped curve to the passive side after 500 cycles. However, the location of this 'zero' particle displacement boundary contour in contact with the pile remained nearly at the same location. This has helped to identify the expansion of the zones of affected soil during the cyclic loading. This expansion is also due to the transfer of contact force from the pile movement, which is a similar phenomenon to the spread of 


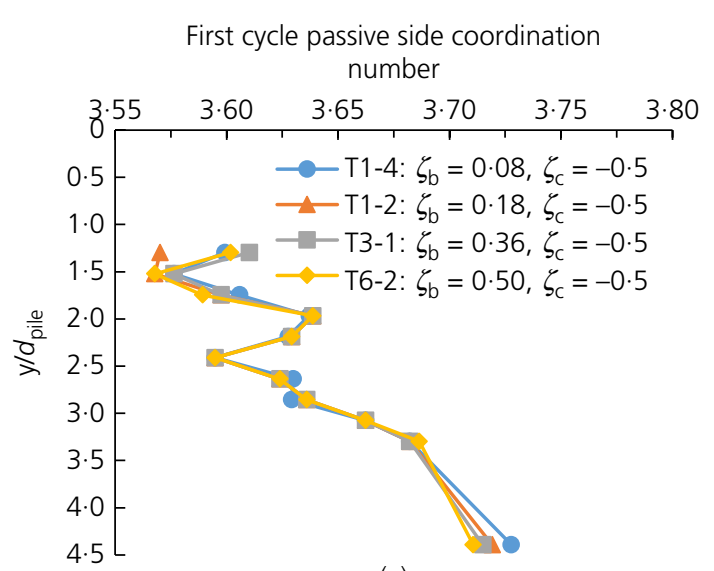

(a)

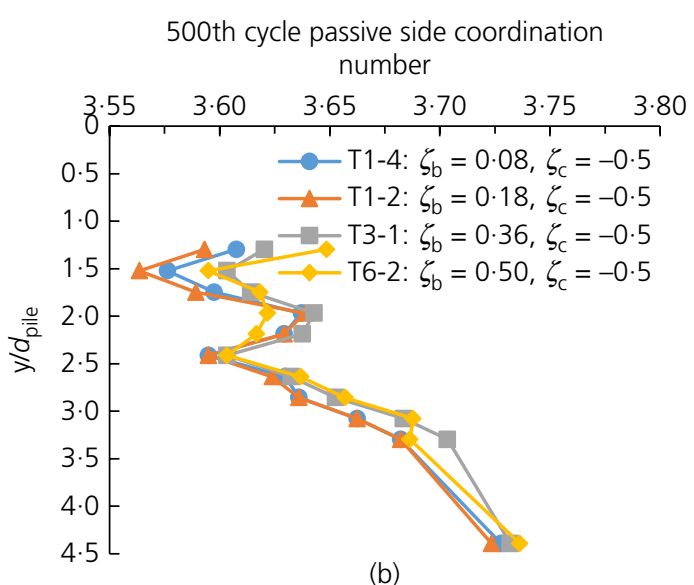

(b)

Figure 13. Effect of $\zeta_{b}$ on the average coordination number of particles on the passive side of the monopile $\left(\zeta_{c}=-0 \cdot 50\right)$ : (a) $N=1$; (b) $N=500$

stress fan (SOF) in the lower bound theory. Figure 13 shows the distributions of the coordination number (defined as the average number of surrounding particles in contact with each particle near the pile) along the pile for the first and 500th cycles. The larger the coordination number, the denser the soil. No significant change of packing density is found in the first cycle, in particular around the pile rotation centre, as can be seen in Figure 13(a). However, densification becomes obvious after 500 cycles, and is the most significant when $\zeta_{\mathrm{b}}=0 \cdot 50$, as shown in Figure 13(b). Also, although more densification appears near the soil surface, there is still densification around and below the pile rotation centre.

\subsubsection{Effect of $\zeta_{c}$}

Figure 14 shows the four cases with the same $\zeta_{\mathrm{b}}(=0 \cdot 36)$, but $\zeta_{\mathrm{c}}$ varies from one-way loading to two-way loading from case T1-4 to T6-2. Because of the same $\zeta_{\mathrm{b}}$, the distributions of Figures 14(a)-14(d) are almost the same, and smaller $\zeta_{\mathrm{c}}$ causes larger displacement. This trend is also seen in Figure 11. As mentioned above, negative $\zeta_{\mathrm{c}}$ means that the cyclic loading is two-way, and positive $\zeta_{\mathrm{c}}$ means one-way loading. Comparing the displacements in these figures, the two-way is obviously larger than the one-way.

After 500 cycles (see Figures 14(e)-14(h)), the area with displacements in the range from -0.05 to $-0.1 \mathrm{~mm}$ is smaller than that in the range from -0.6 to $-1.2 \mathrm{~mm}$. This means that the movements of particles near the pile increase. From the area near the pile tip in Figures 14(e)-14(h), it can be found that the phenomenon of SOF is shrinking during the cyclic test. However the trend is the opposite for the upper area near the pile. At the same time, when the value of $\zeta_{\mathrm{c}}$ is small (i.e. negative), the 'zero' value line inclines significantly. This change is more obvious in Figures 14(i)-14(1) than in Figures 12(i)-12(1). Figure 15 shows that the degree of densification of soil around the pile for one-way loading is generally less than that for two-way loading.

\section{Conclusions}

Numerical DEM simulations of a rigid monopile subject to lateral cyclic loading were performed to compare with data of centrifuge testings. The cyclic displacement accumulation was measured up to 500 load cycles. These cyclic load tests included a range of load amplitudes that increased gradually throughout the test. The low-level cyclic loads were chosen to represent the normal working condition on a monopile, while the larger amplitudes represent extreme working conditions, such as storms and strong ocean currents. For the numerical simulation, the frequency was also a very important factor, and $40 \mathrm{~Hz}$ was chosen for its good match with experimental results.

The main contributions of this paper are listed below.

- The first ten cycles had a strong influence on the cyclic responses of a rigid monopile under cyclic loading;

- There was a close relationship between the accumulated displacement and the cyclic load ratio and amplitude. The impact of load amplitude was obvious. However, the cyclic load ratio was found to control the change of displacement. This was revealed by having significant differences in the influence zones in the particle displacement fields.

- The normalised lateral displacement decreased when the cyclic load ratio increased because one-way loading induced notably less displacement and densification on the passive side than two-way loading. However, normalised lateral displacement increased with load amplitude. 


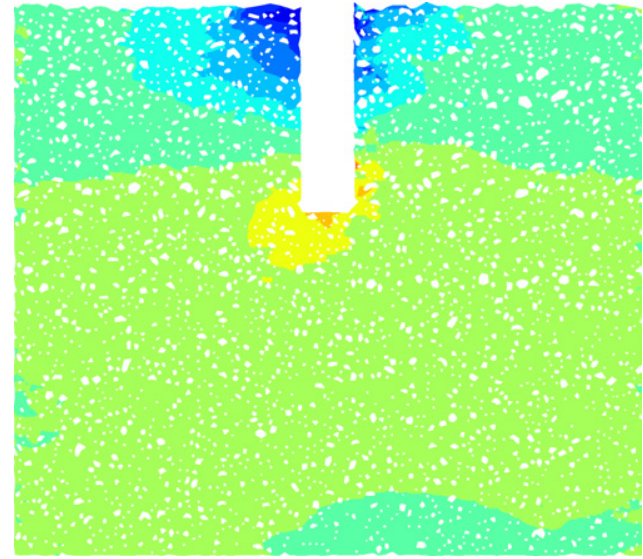

(a)

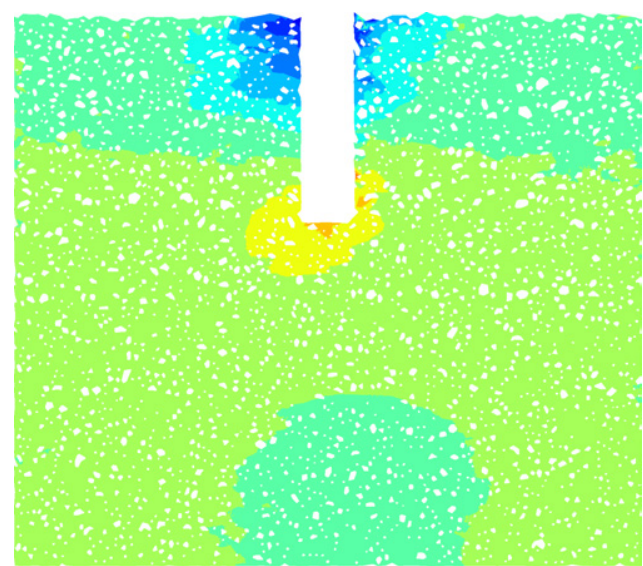

(c)

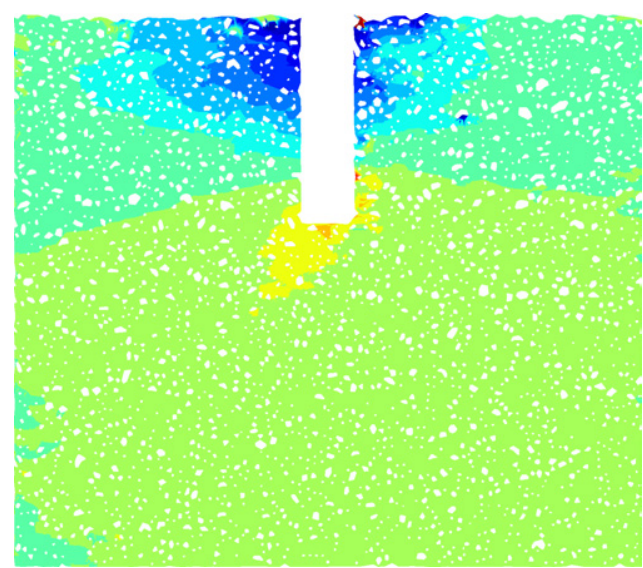

(e)
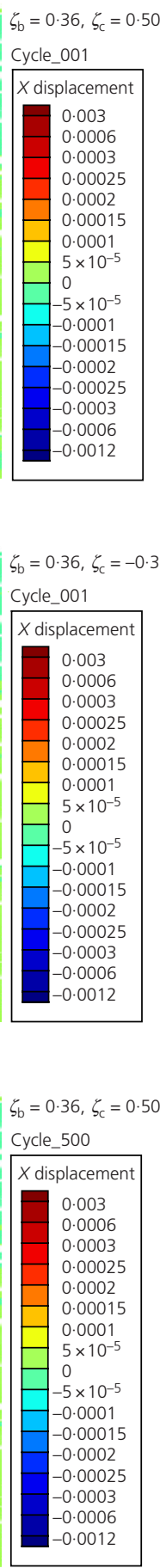

Figure 14. Effect of $\zeta_{c}$ on the accumulated particle horizontal displacement field $\left(\zeta_{b}=0.36\right)$ : (a) $\zeta_{c}=0.50(N=1) ;(b) \zeta_{c}=0.00(N=1)$; (c) $\zeta_{c}=-0.37(N=1) ;(d) \zeta_{c}=-0.50(N=1) ;(e) \zeta_{c}=0.50(N=500) ;(f) \zeta_{c}=0.00(N=500) ;(g) \zeta_{c}=-0.37(N=500) ;(h) \zeta_{c}=-0.50(N=500) ;$ (i) $\zeta_{c}=0.50$ (normalised); (j) $\zeta_{c}=0.00$ (normalised); (k) $\zeta_{c}=-0.37$ (normalised); (I) $\zeta_{c}=-0.50$ (normalised) (continued on next page)

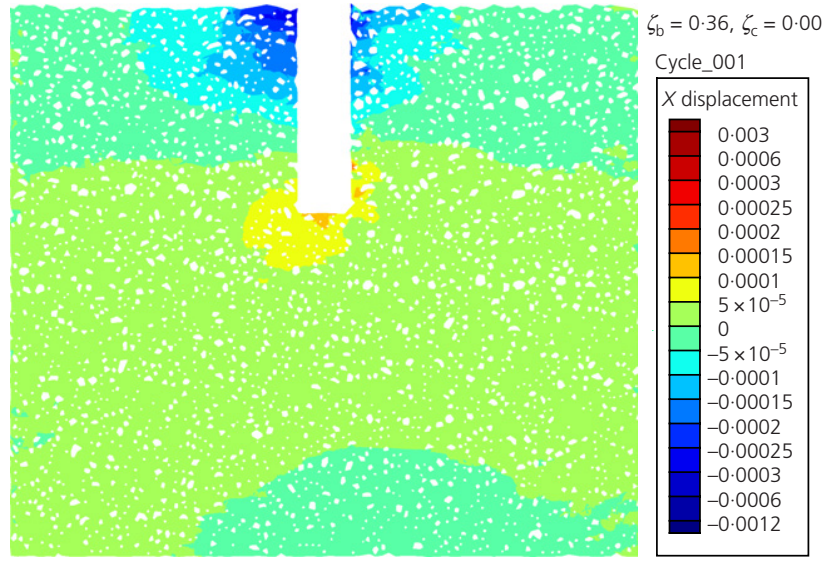

(b)
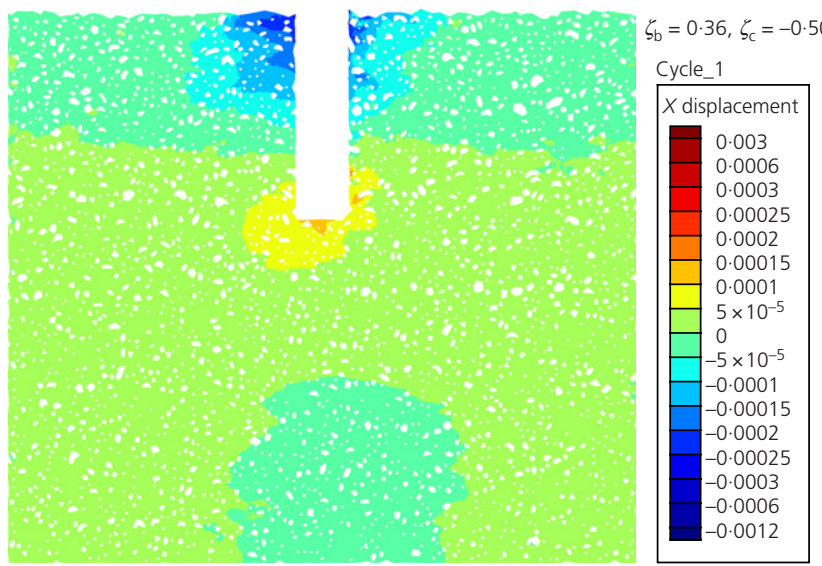

(d)
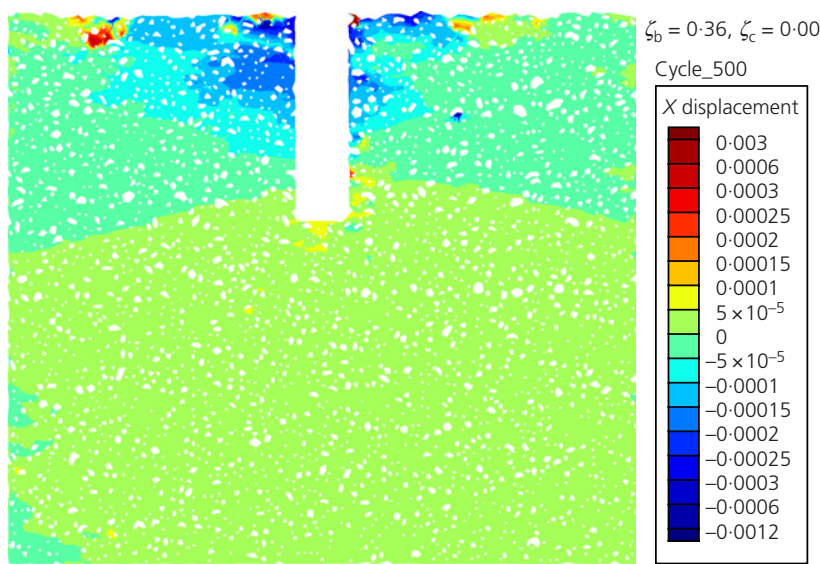

(f)
Under the same load amplitude, both the first cycle secant unloading stiffness $k_{\mathrm{a} 1}$ and the tenth cycle secant unloading stiffness $k_{\mathrm{a} 10}$ increased when the cyclic load ratio increased. Nonetheless, the value of the stiffness ratio $k_{\mathrm{a} 10} / k_{\mathrm{a} 1}$ decreased dramatically. The change of stiffness was more obvious with a larger cyclic load ratio because of the significantly different stiffness observed in the first cycle. 


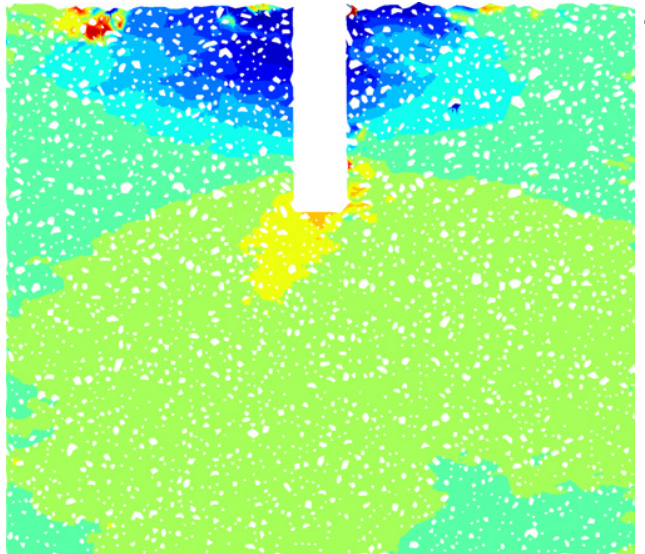

(g)

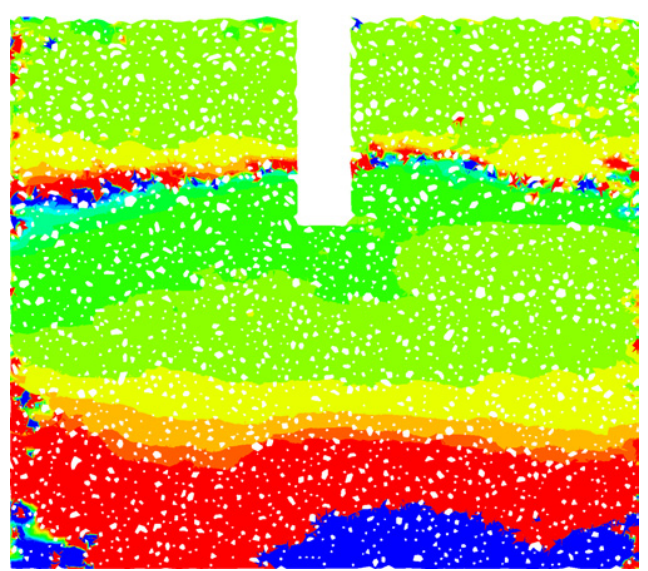

(i)

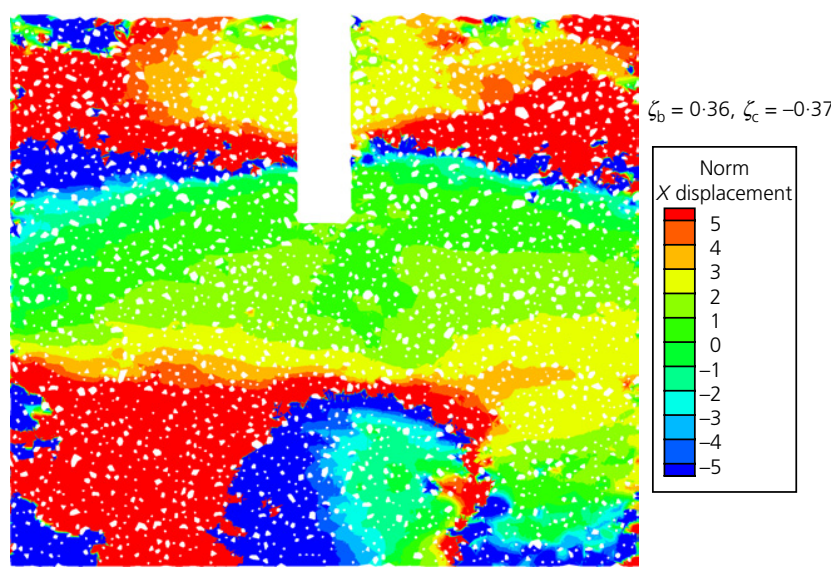

(k)
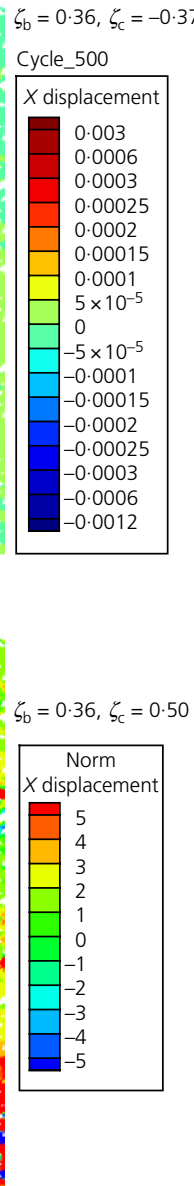
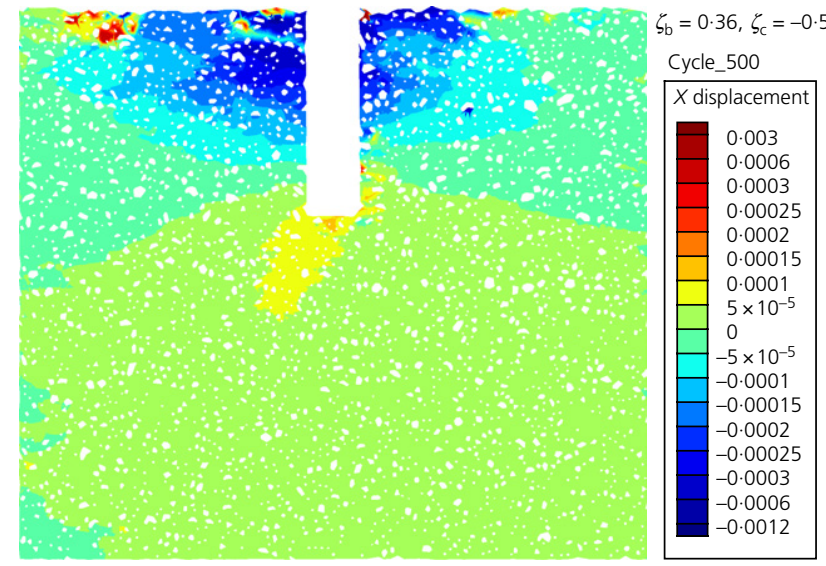

(h)

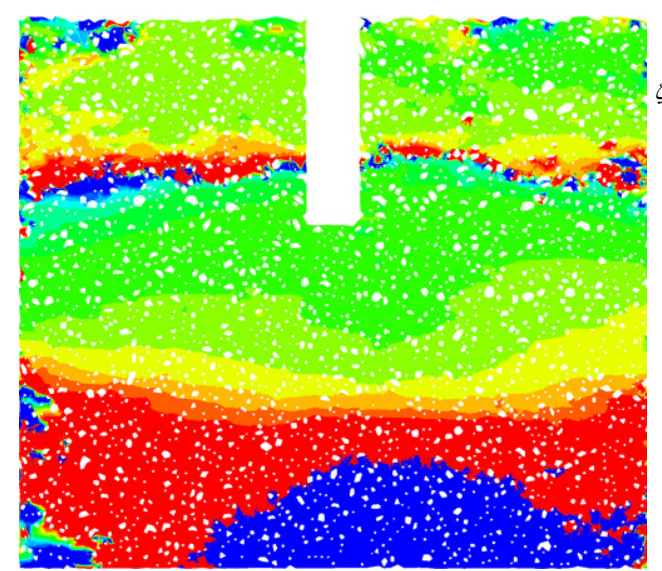

(j)

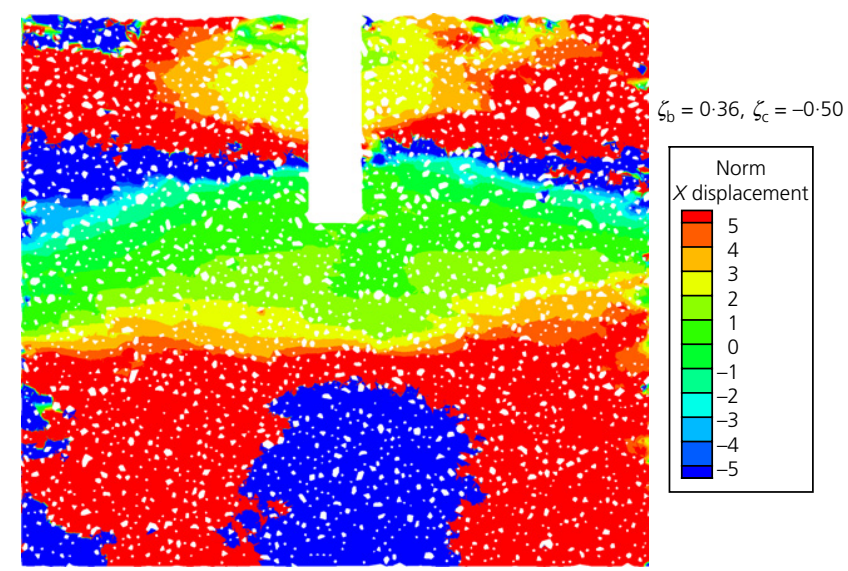

(I)

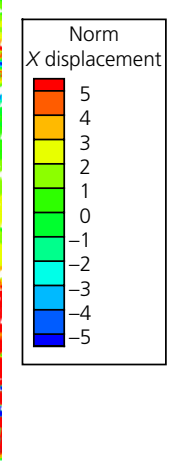

$\zeta_{b}=0.36, \zeta_{c}=0.00$

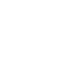




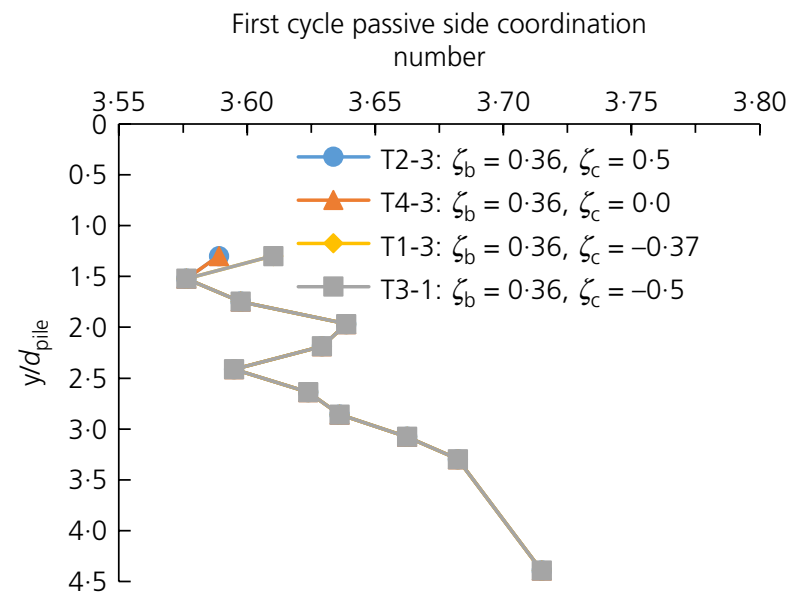

(a)

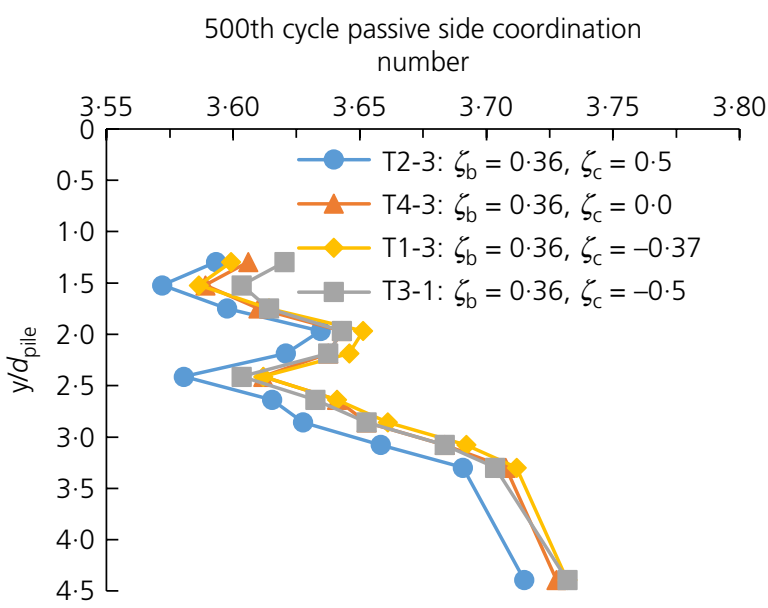

(b)

Figure 15. Effect of $\zeta_{c}$ on the average coordination number of particles on the passive side of the monopile $\left(\zeta_{b}=0 \cdot 36\right)$ : (a) $N=1$; (b) $N=500$

\section{REFERENCES}

Achmus M, Albiker J and Abdel-Rahman K (2010) Investigations on the behavior of large diameter piles under cyclic lateral loading. In Frontiers in Offshore Geotechnics II (Gourvenec S and White D (eds)). CRC Press, Boca Raton, FL, USA, pp. $471-476$

Arany L, Bhattacharya S, Macdonald JH and Hogan SJ (2016) Closed form solution of Eigen frequency of monopile supported offshore wind turbines in deeper waters incorporating stiffness of substructure and SSI. Soil Dynamics and Earthquake Engineering 83: 18-32, http://doi.org/10.1016/j.soildyn.2015.12.011.

Arshad M and O'Kelly BC (2013) Offshore wind-turbine structures: a review. Proceedings of the Institution of Civil Engineers - Energy 166(4): 139-152, http://dx.doi.org/10.1680/ener.12.00019.

Bhattacharya S, Nikitas N, Garnseyc J et al. (2013) Observed dynamic soil-structure interaction in scale testing of offshore wind turbine foundations. Soil Dynamics and Earthquake Engineering 54: 47-60, http://doi.org/10.1016/ j.soildyn.2013.07.012.

Chen RP, Sun YX, Zhu B and Guo WD (2015) Lateral cyclic pile-soil interaction studies on a rigid model monopile. Proceedings of the Institution of Civil Engineers - Geotechnical Engineering 168(2): 120-130, http://dx.doi.org/10.1680/geng.14.00028.

Cuéllar P (2011) Pile Foundations for Offshore Wind Turbines: Numerical and Experimental Investigations on the Behaviour under Short-Term and Long-Term Cyclic Loading. University of Technology Berlin, Berlin, Germany.

Cui L and Bhattacharya S (2015) Dynamic soil-structure interaction around a monopile supporting a wind turbine. In Geomechanics from Micro to Macro (Soga K, Kumar K, Biscontin G and Kuo M (eds)). CRC Press, London, UK, vol. 1, pp. 335-340.

Cui L and Bhattacharya S (2016) Soil-monopile interactions for offshore wind turbines. Proceedings of the Institution of Civil Engineers - Engineering and Computational Mechanics 169(4): 171-182, http://dx.doi.org/10.1680/jencm.16.00006.

Duan N and Cheng YP (2015) Mechanical characteristics of monopile foundation in sand for offshore wind turbine. Proceedings of the 5rd International Workshop of Young Doctors in Geomechanics, Paris, France, pp. 155-161.
Duan N and Cheng YP (2016) A modified method of generating specimens for 2D DEM centrifuge model. Proceedings of GEO-CHICAGO 2016: Sustainable Materials and Resource Conservation, Chicago, IL, USA (Farid A and De A, Reddy KR, Yesiller N, Zekkos D (eds)). American Society of Civil Engineers, Reston, VA, USA, pp. 610-620.

Guo Z, Yu LQ, Wang LZ, Bhattacharya S and Nikitas G (2015) Model tests on the long-term dynamic performance of offshore wind turbines founded on monopiles in sand. Journal of Offshore Mechanics and Arctic Engineering 137(4): 041902.

Houlsby GT (2016) Interactions in offshore foundation design. Géotechnique 66(10): 791-825.

Kirkwood P and Haigh S (2013a) Centrifuge testing of monopiles for offshore wind turbines. In Proceedings of the 23rd International Offshore and Polar Engineering Conference (ISOPE), Anchorage, $A K, U S A$ (Chung JS, Langen I, Kokkinus T and Wang AM (eds)) International Society of Offshore and Polar Engineers, Cupertino, CA, USA, pp. 126-130.

Kirkwood PB and Haigh SK (2013b) Centrifuge testing of monopiles subject to cyclic lateral loading. In Proceedings of 8th International Conference on Physical Modelling in Geotechnics (ICPMG2014), Perth, Australia (Gaudin C and White D (eds)). CRC Press, London, UK, vol. 2, pp. 827-832.

Klinkvort RT (2013) Centrifuge Modelling of Drained Lateral Pile - Soil Response: Application for Offshore Wind Turbine Support Structures. Technical University of Denmark, Kongens Lyngby, Denmark.

Leblanc C (2009) Design of Offshore Wind Turbine Support Structures: Selected Topics in the Field of Geotechnical Engineering. $\mathrm{PhD}$ thesis, Department of Civil Engineering, Aalborg University, Aalborg, Denmark.

Leblanc C, Houlsby GT and Byrne BW (2010) Response of stiff piles in sand to long-term cyclic lateral loading. Géotechnique 60(2): 79-90.

Li Z, Haigh SK and Bolton MD (2010) Centrifuge modelling of mono-pile under cyclic lateral loads. In Proceedings of 7 th International Conference on Physical Modelling in Geotechnics, Zurich, Switzerland (Springman S, Laue J and Seward L (eds)). CRC Press, Taylor \& Francis Group, Boca Raton, FL, USA, vol. 2, pp. 965-970. 
Lin S and Liao J (1999) Permanent strains of piles in sand due to cyclic lateral loads. Journal of Geotechnical and Geoenvironmental Engineering 125(9): 798-802.

Long J and Vanneste G (1994) Effects of cyclic lateral loads on piles in sand. Journal of Geotechnical Engineering 120(1): 225-244.

Nikitas G, Vimalan NJ and Bhattacharya S (2016) An innovative cyclic loading device to study long term performance of offshore wind turbines. Soil Dynamics and Earthquake Engineering 82: 154-160, http://doi.org/10.1016/j.soildyn.2015.12.008.

Peralta P and Achmus M (2010) An experimental investigation of piles in sand subjected to lateral cyclic loads. In Proceedings of 7 th International Conference on Physical Modelling in Geotechnics, Zurich, Switzerland (Springman S, Laue J and Seward L (eds)). CRC Press, Boca Raton, FL, USA, vol. 1, pp. 985-990.
Randolph MF (1981) The response of flexible piles to lateral loading. Géotechnique 31(2): 247-259.

Rosquoet F, Thorel L, Garnier J and Canepa Y (2007) Lateral cyclic loading of sand-installed piles. Soils and Foundations 47(5): 821-832. Schofield AN (1980) Cambridge geotechnical centrifuge operations. Géotechnique 30(3): 227-268.

Yu LQ, Wang LZ, Guo Z et al. (2015) Long-term dynamic behavior of monopile supported offshore wind turbines in sand. Theoretical and Applied Mechanics Letters 5(2): 80-84.

Zania V and Hededal O (2012) Friction effects on lateral loading behavior of rigid piles. In Proceedings GeoCongress 2012 (Hryciw RD, Athanasopoulos-Zekkos and Yesiller N (eds)). American Society of Civil Engineers, Reston, VA, USA, pp. 366-375.

\section{How can you contribute?}

To discuss this paper, please email up to 500 words to the editor at journals@ice.org.uk. Your contribution will be forwarded to the author(s) for a reply and, if considered appropriate by the editorial board, it will be published as discussion in a future issue of the journal.

Proceedings journals rely entirely on contributions from the civil engineering profession (and allied disciplines). Information about how to submit your paper online is available at www.icevirtuallibrary.com/page/authors, where you will also find detailed author guidelines. 